\title{
Making cobordisms symplectic
}

\author{
Yakov Eliashberg* Emmy Murphy \\ Stanford University Northwestern University
}

\begin{abstract}
We establish an existence $h$-principle for symplectic cobordisms of dimension $2 n>4$ with concave overtwisted contact boundary.
\end{abstract}

\section{Introduction}

\section{Symplectic cobordisms}

We say that $\left(W, \omega, \xi_{-}, \xi_{+}\right)$is a symplectic cobordism between contact manifolds $\left(\partial_{ \pm} W, \xi_{ \pm}\right)$if

- $W$ is a smooth cobordism between $\partial_{-} W$ and $\partial_{+} W$, and

- $\omega$ is a symplectic form which admits a Liouville vector field $Z$ near $\partial W$ such that $Z$ is inwardly transverse to $\partial_{-} W$, outwardly transverse to $\partial_{+} W$ and the contact forms $\lambda_{ \pm}=\left.\iota(Z) \omega\right|_{\partial_{ \pm} W}$ define the contact structures $\xi_{ \pm}$.

All cobordisms we consider in this paper are assumed to be connected while their boundaries $\partial_{ \pm} W$ could be disconnected. We recall that a vector field $Z$ is called Liouville for a symplectic form $\omega$ if $d(\iota(Z) \omega)=\omega$.

By a Liouville cobordism $\left(W, \lambda, \xi_{-}, \xi_{+}\right)$between contact manifolds $\left(\partial_{ \pm} W, \xi_{ \pm}\right)$we mean a symplectic cobordism $(W, \omega)$ between $\left(\partial_{ \pm} W, \xi_{ \pm}\right)$with a fixed primitive $\lambda$,

\footnotetext{
*Partially supported by the NSF grants DMS-1505910 and DMS-1807270

${ }^{\dagger}$ Partially supported by the NSF grant DMS-1906564
} 
$d \lambda=\omega$, so that the Liouville vector field $Z$ (defined by being the $\omega$-dual to $\lambda$ ) is inwardly transverse to $\partial_{-} W$ and outwardly transverse to $\partial_{+} W$.

An obvious necessary condition for finding a symplectic cobordism structure between contact manifolds $\left(\partial_{-} W, \xi_{-}\right)$and $\left(\partial_{+} W, \xi_{+}\right)$on a fixed smooth cobordism $W$ is the existence of an almost symplectic cobordism structure; that is a non-degenerate but not necessarily closed 2-form $\eta$ on $W$ which coincides with $d \lambda_{ \pm}$near $\partial_{ \pm} W$.

We call such $\eta$ an almost symplectic cobordism structure. The homotopy class of an almost symplectic structure is determined by the homotopy class of an almost complex structure $J$, such that $J$ is tamed by $\eta$, and $\xi_{ \pm}$are $J$-complex subbundles.

Note that almost symplectic forms $\eta$ and $f \eta$, where a $f$ is a positive function, are canonically homotopic. Hence, given two almost symplectic form $\eta$ and $\widetilde{\eta}$ on a manifold $M$ which coincide on $\left.T M\right|_{A}$ for a closed subset $A \subset M$, we say, slightly abusing the terminology, that $\eta$ and $\tilde{\eta}$ are homotopic relative $A$ if they can be connected by a homotopy of almost symplectic forms $\eta_{t}$ such that $\left.\eta_{t}\right|_{\left.T M\right|_{A}}=\left.f_{t} \eta\right|_{\left.T M\right|_{A}}$ where $f_{t}: A \rightarrow \mathbb{R}$ is family of positive functions.

The following theorem is the main result of the paper:

Theorem 1.1. Let $(W, \eta)$ be an almost symplectic cobordism of dimension $2 n>2$ between non-empty contact manifolds $\left(\partial_{ \pm} W, \xi_{ \pm}\right)$. Suppose that

- the contact structure $\xi_{-}$is overtwisted on at least one of the connected components of $\partial_{-} W$;

- if $n=2$ the contact structure $\xi_{+}$is overtwisted on at least one of the connected components of $\partial_{+} W$.

Then there exists a Liouville cobordism structure $\left(W, \lambda, \xi_{-}, \xi_{+}\right)$such that $d \lambda$ and $\eta$ are homotopic as almost symplectic structures fixed on $\partial W$.

As Proposition 1.10 below shows, the condition that the positive end of a 4-dimensional cobordism has an overtwisted component is essential. On the other hand, Theorem 1.1 implies

Corollary 1.2. Let $W$ be a 4-dimensional manifold with non-empty boundary $\partial W$, and let $\eta$ be an almost symplectic form such that $\left.\eta\right|_{\mathcal{O} p} \partial W=d \lambda$ for a Liouville form $\lambda$ whose Liouville field is outwardly transverse to $\partial W$. Then the (interior) connected sum $W^{\prime}:=W \#\left(S^{3} \times[0,1]\right)$ admits a Liouville cobordism structure $\Lambda$ with $\partial_{+} W^{\prime}:=$ $\partial W \amalg\left(S^{3} \times 1\right), \partial_{-} W=S^{3} \times 0$ and $\left.\Lambda\right|_{\mathcal{O}_{p} \partial W}=\lambda$. 
Remark 1.3. Note that any two odd-dimensional manifolds which are endowed with a stable almost complex structures are cobordant in an almost complex category, see [21, 25]. In particular, for any contact manifolds $\left(\partial_{ \pm} W, \xi_{ \pm}\right)$an almost symplectic cobordism $(W, \eta)$, as in Theorem 1.1 , always exists.

Any stable almost complex structure on an odd-dimensional manifold is realized by an overtwisted contact structure, see [1]. Thus the above theorem implies that any smooth cobordism $W$ with non-empty boundaries that admits an almost symplectic structure also admits a structure of a symplectic cobordism between two contact manifolds.

The notion of an overtwisted contact structure was introduced in [4] in dimension 3 and extended to all dimensions in [1]. Without giving precise definitions, which will be not important for the purposes of this paper, we summarize below the main results about overtwisted contact structures which will be used in this paper. Theorem 1.4 is proven in [1] for $n>1$ and in [4] for $n=1$. Theorem 1.5 is proven in [2]. If $\xi=\{\alpha=0\}$ is an overtwisted contact structure, we will refer to $\alpha$ as an overtwisted contact form.

All contact structures which appear in this paper will be coorientable. We recall that a cooriented almost contact structure is a cooriented hyperplane field $\zeta \subset T M$ equipped with a linear symplectic structures $\eta$ on it, which is defined up to a positive conformal factor. Cooriented almost contact structures can be represented by pairs $(\alpha, \eta)$ where $\alpha$ is a non-vanishing 1 -form on $M$ and $\eta$ is a non-degenerate two-form on the hyperplane field $\xi=\{\alpha=0\}$. We note that given a maximal $(=\operatorname{dim} M-1)$ rank 2-form $\eta$ on $M$ the corresponding 1-form $\alpha$ can be reconstructed uniquely up to a contractible choice. The existence of an almost contact structure is equivalent to the existence of a stable almost complex structure on $M$, i.e. a complex structure on the bundle $T M \oplus \varepsilon^{1}$ where $\varepsilon^{1}$ is the trivial line bundle over $M$.

Theorem 1.4. Let $\theta$ be a cooriented almost contact structure on a $(2 n+1)$-dimensional manifold $M$ which is genuinely contact on a neighborhood $\mathcal{O} p$ A of a closed subset $A \subset M$. Then there exists an overtwisted contact structure $\xi$ on $M$ which coincides with $\theta$ on a neighborhood of $A$ and which is homotopic to $\eta$ through almost contact structures fixed on $A$. Moreover, suppose we are given two contact structures $\xi_{0}, \xi_{1}$ and a homotopy $\eta_{t}, t \in[0,1]$, of almost contact structures such that

- $\xi_{0}, \xi_{1}$ and $\eta_{t}$ coincide on $\mathcal{O} p$ A for all $t \in[0,1]$,

- $\xi_{0}$ and $\xi_{1}$ are overtwisted on every connected component of $M \backslash A$.

Then there exist 
- a homotopy $\xi_{t}, t \in[0,1]$, of genuine contact structures connecting $\xi_{0}$ and $\xi_{1}$, and

- a 2-parametric family $\Theta_{t, s}$ of almost contact structures

such that

- $\Theta_{t, 0}=\xi_{t}, \Theta_{t, 1}=\theta_{t}, \Theta_{0, s}=\xi_{0}$ and $\Theta_{1, s}=\xi_{1}$ for all $t, s \in[0,1]$.

- $\left.\Theta_{t, s}\right|_{\mathcal{O} p A}=\left.\xi_{0}\right|_{\mathcal{O} p}$.

Theorem 1.5. Let $(M,\{\alpha=0\})$ be an overtwisted contact manifold and $\left(D_{R}, \mu\right)$ the disc of radius $R$ in $\mathbb{R}^{2}$ endowed with a Liouville form $\mu=x d y-y d x$. Then for sufficiently large values of $R$, the product $\left(M \times D_{R},\{\alpha \oplus \mu=0\}\right)$ is also overtwisted.

We note that any contact manifold of dimension $>1$ can be made overtwisted without changing its almost contact homotopy class by a modification in a neighborhood of one of its points, and that any two definitions of overtwistedness for which Theorem 1.4 holds are equivalent.

For any closed form $\omega$ on $W$ equal to $d \lambda_{ \pm}$on $\partial_{ \pm} W$ one can canonically associate a cohomology class $[\omega] \in H^{2}(W, \partial W)$. Indeed, take any 1-form $\lambda$ on $W$ extending $\lambda_{ \pm}$ Then the cohomology class of $[\omega-d \lambda] \in H^{2}(W, \partial W)$ is independent of the choice of the extension $\lambda$.

Corollary 1.6. Under the assumptions of Theorem 1.1 there is a symplectic cobordism structure $\omega$ on $W$ between $\xi_{-}$and $\xi_{+}$so that $[\omega]$ is equal to any given cohomology class $a \in H^{2}(W, \partial W)$.

Indeed, let $\lambda$ be the Liouville form provided by Theorem 1.1, and let $\sigma$ be any closed form with compact support representing $a \in H^{2}(W, \partial W)$. Then for a sufficiently large constant $C$ the form $\omega=C d \lambda+\sigma$ is symplectic and has the required properties.

It was proven in [2], see also Corollary 2.12 below, that if $n>2$ then for any contact structure $\xi$ on $\partial_{+} W$ there is a Liouville concordance (i.e. a Liouville cobordism diffeomorphic to $\left.\partial_{+} W \times[0,1]\right)$ between $\xi$ on the positive end and an overtwisted contact structure $\xi_{\text {ot }}$ on the negative end. This is not the case for $n=2$, as Proposition 1.10 below demonstrates. Hence, if $n>2$ it is sufficient to prove Theorem 1.1 for the case when both contact structures $\xi_{ \pm}$are overtwisted. This is the only reason why we need an additional hypothesis when $n=2$. 


\section{Symplectic manifolds with a conical singularity}

Given a $2 n$-dimensional manifold $X$ with boundary $\partial X$, a symplectic form $\omega$ on $X \backslash p$, $p \in X$, and a contact structure $\xi$ on $\partial X$, we say that $(X, \omega, \xi)$ is a symplectic domain with a conical singularity at $p$ and contact boundary $(\partial X, \xi)$ if $(\partial X, \xi)$ is a positive contact boundary in the above sense, and near $p$ there exists a Liouville field $Z$ and a Morse function $\phi$ with the minimum at $p$ such that $d \phi(Z)>0$. In other words, in a punctured ball centered at $p$ the form $\omega$ is symplectomorphic to the negative part of the symplectization of a contact structure $\zeta$ on the boundary sphere $S^{2 n-1}$. We will call $\left(S^{2 n-1}, \zeta\right)$ the link of the singularity $p$. By removing from $X$ a ball $B$ centered at $p$, we can equivalently view a symplectic structure $\omega$ on $X$ with a conical singularity at $p$ as a symplectic cobordism structure on $\dot{X}=X \backslash \operatorname{Int} B$ between $\left(\partial_{-} \dot{X}:=\partial B, \zeta\right)$ and $\left(\partial_{+} \dot{X}:=\partial X, \xi\right)$.

Of course, if the contact structure $\zeta$ is standard, then the form extends to a nonsingular symplectic form on the whole $X$. Note that if $\zeta$ is overtwisted then according to Theorem $1.4 \zeta$ is uniquely determined up to isotopy by the homotopy class of $\omega$ in the space of non-degenerate 2 -forms on a punctured neighborhood of $p$.

The following result is a special case of Theorem 1.1 .

Theorem 1.7. Let $X$ be a compact $2 n$-dimensional manifold with non-empty boundary $\partial X$, and let $\xi$ a contact structure on $\partial X$. Let $\eta$ be a non-degenerate 2-form on $X \backslash p, p \in X$, which is equal to $d \lambda$ near $\partial X$, where $\lambda$ is any 1-form satisfying $\xi=\left\{\left.\lambda\right|_{\partial X}=0\right\}$ and such that the Liouville vector field dual to $\lambda$ is outward transverse to $\partial X$. Let $a \in H^{2}(X, \partial X)$ be a relative cohomology class. If $n=2$ assume, in addition, that $\xi$ is overtwisted. Then there exists a symplectic structure $\omega$ on $X$ with a conical singularity at $p$ with an overtwisted link $\left(S^{2 n-1}, \zeta\right)$ and positive contact boundary $(\partial X, \xi)$, such that the cohomology class $[\omega] \in H^{2}(X \backslash p, \partial X)=H^{2}(X, \partial X)$ coincides with $a$, and the homotopy class of $\left.\omega\right|_{X \backslash p}$ as a non-degenerate form coincides with the rel. $\partial X$ homotopy class of $\eta$.

Remark 1.8. 1. In contrast with Theorem 1.7, the construction of non-singular symplectic structures on $X$ is severely constrained. For instance, according to Gromov's theorem in dimension 4 and Eliashberg-Floer-McDuff's theorem in higher dimensions, see [16, 18], any symplectic manifold $(X, \omega)$ bounded by the standard contact sphere and satisfying the condition $\left.[\omega]\right|_{\pi_{2}(X)}=0$ has to be diffeomorphic to a ball.

2. It is interesting to compare the flexibility phenomenon for symplectic structures with conical singularities with overtwisted links, exhibited in Theorem 1.7, with a similar flexibility phenomenon for Lagrangian manifolds with conical singularities with loose Legendrian links, see [10]. 
Theorem 1.7 implies the following

Corollary 1.9. Let $X$ be a closed manifold of dimension $2 n>4$ that admits an almost complex structure on $X \backslash p, p \in X$. Let $a \in H^{2}(X)=H^{2}(X \backslash p)$ be any cohomology class. Then for any closed symplectic $2 n$-dimensional manifold $(Z, \omega)$, the connected sum $X \# Z$ admits a symplectic form with a conical singularity in the cohomology class $a+C[\omega]$ for a sufficiently large constant $C>0$.

Proof. Let $B$ be a ball centered at $p$. The obstruction to extending an almost complex structure $J$ from $X \backslash \operatorname{Int} B$ to $X$ is an element $\alpha \in \pi_{2 n-1}(S O(2 n) / U(n))$. Choose two disjoint balls $B_{1}, B_{2} \subset$ Int $B$ and define $J$ on a neighborhood $\mathcal{O} p \partial B_{1}$ of $\partial B_{1}$ as the push-forward of the standard complex structure on the boundary of a ball $D \subset \mathbb{C}^{n}$ under an orientation preserving diffeomorphism of $h: \mathcal{O} p \partial D \rightarrow \mathcal{O} p \partial B_{1}$ such that $h(\partial D)=B_{1}$, and $h$ sends the outward normal vector field to the boundary $D$ to the inward normal vector field to the boundary of $B_{1}$. Note that $J$ does not extend to an almost complex structure on $B_{1}$, unless $n=1,3$. Let $\beta \in \pi_{2 n-1}(S O(2 n) / U(n))$ be the obstruction to this extension. We furthermore extend $J$ to $B \backslash \operatorname{Int}\left(B_{1} \cup B_{2}\right)$ and note that the obstruction to extending $J$ to $B_{2}$ is equal to $\alpha-\beta$. By construction the almost complex structure $J$ on $\mathcal{O} p \partial B_{1}$ is compatible with the push-forward $h_{*} \xi_{\text {std }}$ of the standard contact structure $\xi_{\text {std }}$ on $\partial D$, co-oriented by the outward normal. Next, we apply Theorem 1.7 to get a symplectic form $\omega_{X}$ on $X \backslash \operatorname{Int} B_{1}$ with a conical singularity at the center of $B_{2}$, so that $\left[\omega_{X}\right]=a$ and $\partial_{+}\left(X \backslash \operatorname{Int} B_{1}, \omega_{X}\right) \cong\left(S^{2 n-1}, \xi_{\text {std }}\right)$. Then $\left(X \backslash \operatorname{Int} B_{1}, \omega_{X}\right)$ can be implanted into the symplectic manifold $Z$, after rescaling the symplectic form of $Z$ if necessary.

As we already pointed out above, when $n=2$, the assumption that at least one of components of the positive boundary is overtwisted is essential, as the following proposition proven in [23, 17] demonstrates:

Proposition 1.10. Suppose $\left(W, \omega, \xi_{-}, \xi_{+}\right)$is an exact 4-dimensional symplectic cobordism and the contact structure $\xi_{-}$is symplectically fillable. Then there is no exact symplectic cobordism structure $\left(W, \widetilde{\omega}, \widetilde{\xi}_{-}, \xi_{+}\right)$where $\omega$ and $\widetilde{\omega}$ are in the same homotopy class of almost symplectic forms and $\widetilde{\xi}_{-}$is overtwisted. In particular, for any fillable contact manifold $(M, \xi)$ there is no symplectic concordance $(M \times[-1,1], \omega)$ in either direction between $(M, \xi)$ and $(M, \widetilde{\xi})$ with overtwisted $\widetilde{\xi}$.

Note that the non-existence of a symplectic cobordism between a fillable contact structure on the negative end, and an overtwisted structure on the positive one is a universal fact which holds for all symplectic cobordisms in all dimensions, see [16, 6, 26, 1]. 
We do not know whether the condition $\partial_{+} W \neq \varnothing$ in Theorem 1.1 is essential when $n>2$. We note, however, that Theorem 1.1 implies that every overtwisted contact manifold $(M, \xi)$ of dimension $>3$ admits a symplectic cap, i.e. there exists a symplectic cobordism $(W, \omega)$ with $\partial_{+} W=\varnothing$ and $\partial_{-}(W, \omega)=(M, \xi)$. Indeed, the group of complex bordisms is trivial in odd dimensions, see [21, 25]. Hence, according to Theorem 1.1 there is a symplectic cobordism between $(M, \xi)$ on the negative end and the standard contact sphere on the positive one, which then can be capped by any closed symplectic manifold, as in the proof of Corollary 1.9. An existence of a symplectic cap for all (and in particular) overtwisted contact 3-manifolds was originally proven in [12], see also [8] and [11]. However, these papers do not give much on the topology of the symplectic cap.

\section{Conformal symplectic manifolds}

A conformal symplectic structure on a manifold $M$ is given by an atlas of symplectic charts $\left(U_{i}, \omega_{i}\right)$, such that the transition maps $f_{i j}$ are conformally symplectic, i.e. $f_{i j}^{*} \omega_{i}=c_{i j} \omega_{j}$ for positive constants $c_{i j} \in \mathbb{R}$.

Equivalently, a conformal symplectic structure can be defined as a symplectic structure with coefficients in a flat principal bundle with fiber the multiplicative group $\mathbb{R}_{+}$of positive real numbers; such a bundle can be described by a representation $\theta: \pi_{1}(M) \rightarrow \mathbb{R}_{+}$. In other words, a conformal symplectic structure on $M$ is a symplectic structure $\omega$ on the universal cover $\widehat{M}$ of $M$ such that the action of $\pi_{1}(M)$ by deck transformations on $\widehat{M}$ satisfies $g^{*} \omega=\theta(g) \omega$ for any $g \in \pi_{1}(M)$. The representation $\theta: \pi_{1}(M) \rightarrow \mathbb{R}_{+}$factors through a homomorphism $\bar{\theta}: H_{1}(M, \mathbb{R}) \rightarrow \mathbb{R}_{+}$, so that $\mu:=\log \bar{\theta}: H_{1}(M, \mathbb{R}) \rightarrow \mathbb{R}$ is an additive homomorphism, which therefore defines a cohomology class in $H^{1}(M, \mathbb{R})$ which we will denote by $\mu_{\omega}$. Theorem 1.1 implies the following $h$-principle for conformal symplectic structures.

Theorem 1.11. Let $(M, \eta)$ be a closed $2 n$-dimensional almost symplectic manifold and suppose $\mu \in H^{1}(M, \mathbb{Z})$ is a non-zero cohomology class. Then there exists a conformal symplectic structure $\omega$ in the formal homotopy class of $\eta$, with $\mu_{\omega}=c \mu$ for some real $c \neq 0$.

Proof. There exists a smooth map $f: M \rightarrow S^{1}$ so that $\mu=f^{*} a$, where $a$ is the generator of $H^{1}\left(S^{1}, \mathbb{Z}\right)$. Denote $\Sigma:=f^{-1}(p)$ for a regular value $p$ of $f$. By cutting $M$ open along $\Sigma$ we get a cobordism $W$ with $\partial_{ \pm} W \cong \Sigma$. Let us endow the boundary components $\partial_{ \pm} W$ with copies of the unique overtwisted contact structure $\xi$ determined by the almost complex structure $J$. Theorem 1.1 yields a symplectic 
cobordism structure $\omega$ on $W$ with the prescribed contact boundaries, and in the relative homotopy class determined by $\eta$. We can assume that the contact structures on $\partial_{ \pm} W \cong \Sigma$ are given by contact forms $\alpha_{ \pm}$, such that $\alpha_{+}=k \alpha_{-}$for some constant $k>0$. Hence by identifying the symplectic forms $\left.k \omega\right|_{\mathcal{O}_{p} \partial_{-} W}$ and $\left.\omega\right|_{\mathcal{O}_{p} \partial_{+} W}$ we get a conformal symplectic structure $\bar{\omega}$ on $M$ corresponding to the cohomology class $\mu_{\bar{\omega}}=(\log k) \mu$.

Remark 1.12. M. Bertelson and G. Meigniez informed us that by further developing the methods of this paper they proved in their forthcoming paper a stronger version of Theorem 1.11, establishing existence of a conformal symplectic structure with any prescribed non-zero cohomology class $[\mu] \in H^{1}(M, \mathbb{R})$.

\section{Historical remarks}

The first constructions of symplectic cobordisms between contact manifolds were based on the Weinstein handlebody construction, see [5, 27, 3] and Theorem 2.8 below. Examples of Liouville domains with disconnected contact boundary (and hence non-Weinstein) were constructed in [20] in dimension 4, see also [22]. This yields construction of Liouville domains of dimension $2 n$ with non-trivial homology up to dimension $\sim \frac{3 n}{2}$, see [9]. High-dimensional Liouville domains with disconnected boundary were constructed in [14] and [19]. See [13, 28, 19] for more constructions of non-Weinstein symplectic cobordisms. Related problems concerning (different flavors of) symplectic fillability and the topology of symplectic fillings were extensively studied, especially in the contact 3-dimensional case. See Chris Wendl's blog [29] for a survey and a discussion of related results and problems.

\section{Acknoledgements}

We thank Chris Wendl for providing some of the above references and Nikolai Mishachev for making several pictures. We are also grateful to the anonymous referees for constructive critical remarks and useful suggestions, and to Oleg Lazarev and Dylan Cant for the attentive reading of the manuscript. The authors are grateful to the following institutions for their support and hospitality: RIMS, Kyoto, and ITS ETH, Zurich (the first author), and IAS, Princeton (the second author). 


\section{Topological and symplectic preliminaries}

\subsection{Sutured cobordisms}

Definition. A sutured cobordism is a compact manifold $W$ with boundary with corners together with a vector field $Z$ on $\mathcal{O} p \partial W$ such that

- the boundary $\partial W$ is presented as the union of two manifolds $\partial_{-} W$ and $\partial_{+} W$ with common boundary $\partial^{2} W=\partial_{+} W \cap \partial_{-} W$, along which $\partial W$ has a corner 1 ;

- the vector field $Z$ is inwardly transverse to $\partial_{-} W$ and outwardly transverse to $\partial_{+} W$.

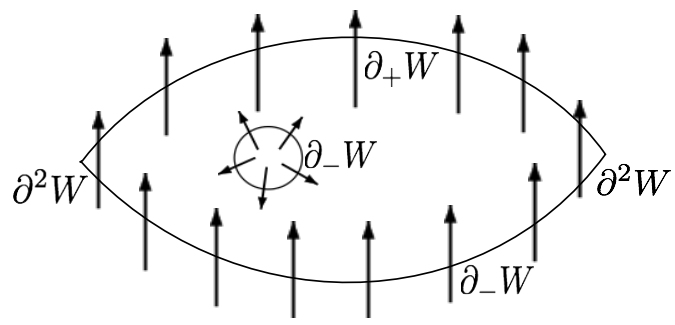

Fig. 2.1: A sutured cobordism $W$. Note that $\partial_{ \pm} W$ may be disconnected. In the figure, $\partial_{-} W$ has one closed component and one component with boundary.

A traditional cobordism between closed manifolds $\partial_{-} W$ and $\partial_{+} W$ is a special case of a sutured cobordism when $\partial^{2} W=\varnothing$. In the rest of the paper all considered cobordisms are allowed to be sutured unless it is stated otherwise.

Remark 2.1. a) The sutured cobordism notion is parallel to the notion of a cobordism between manifolds with boundary, when the cobordism is required to be trivial over the boundary. Such a cobordism can be obtained from a sutured cobordism by shaving off a neighborhood of the corner $\partial^{2} W$ formed by trajectories of the vector field $Z$ originating from an open collar of $\partial\left(\partial_{-} W\right)=\partial^{2} W$ in $\partial_{-} W$, see Figure 2.2.

To go back to a sutured cobordism, one collapses the part $\partial_{-}\left(\partial_{-} W\right) \times[0,1]$ of $\partial W$ into $\partial^{2} W$. In particular given a manifold $N$ with boundary we can associate with the trivial cobordism $N \times[-1,1]$ its sutured version $\hat{N}$ as follows. Choose a smooth

\footnotetext{
${ }^{1}$ i.e. each point of $\partial^{2} W$ has a neighborhood diffeomorphic to $\mathbb{R}^{n-2} \times\left\{(x, y) \in \mathbb{R}^{2} ; x, y \geqslant 0\right\}$, $n=\operatorname{dim} W$.
} 


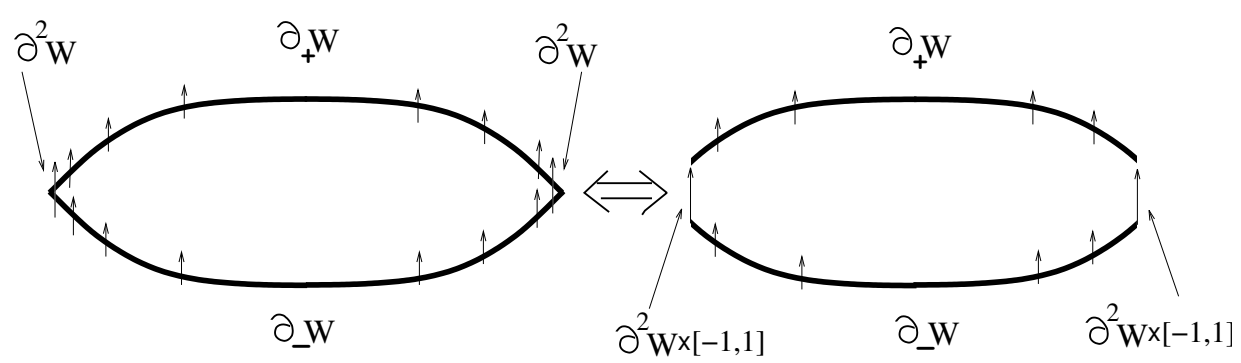

Fig. 2.2: Shaving off the corner of a sutured cobordism

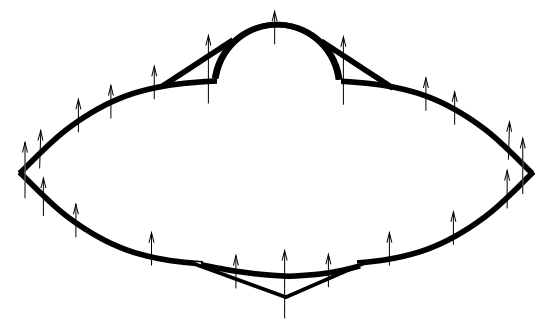

Fig. 2.3: Smoothing non-essential corners of a sutured cobordism.

function $\psi: N \rightarrow[0,1]$ such that $\partial N$ equals the regular level set $\{\psi=0\}$. Define $\widehat{N}:=\{(x, t) \in N \times[-1,1] ;-\psi(x) \leqslant t<\psi(x)$. $\}$. We refer to $\widehat{N}$ as the sutured version of the trivial cobordism $N \times[-1,1]$.

b) The sutured cobordisms which appear in our constructions may sometimes have additional non-essential corners characterized by the property that the corresponding vector field $Z$ is transverse in the same sense (i.e. inwards or outwards) to all boundary faces adjacent to the corner. Such a corner can always be removed by smoothing the boundary $\partial W$ in a neighborhood of the corner, while keeping the boundary faces transverse to $Z$. See Fig. 2.3.

Let us discuss some operations one can perform on sutured cobordisms. We do not specify below the choice of the required vector field $Z$ when it is clear from the context or irrelevant.

Concatenation. Let $W$ and $W^{\prime}$ be two sutured cobordisms and let $P$ be a connected 


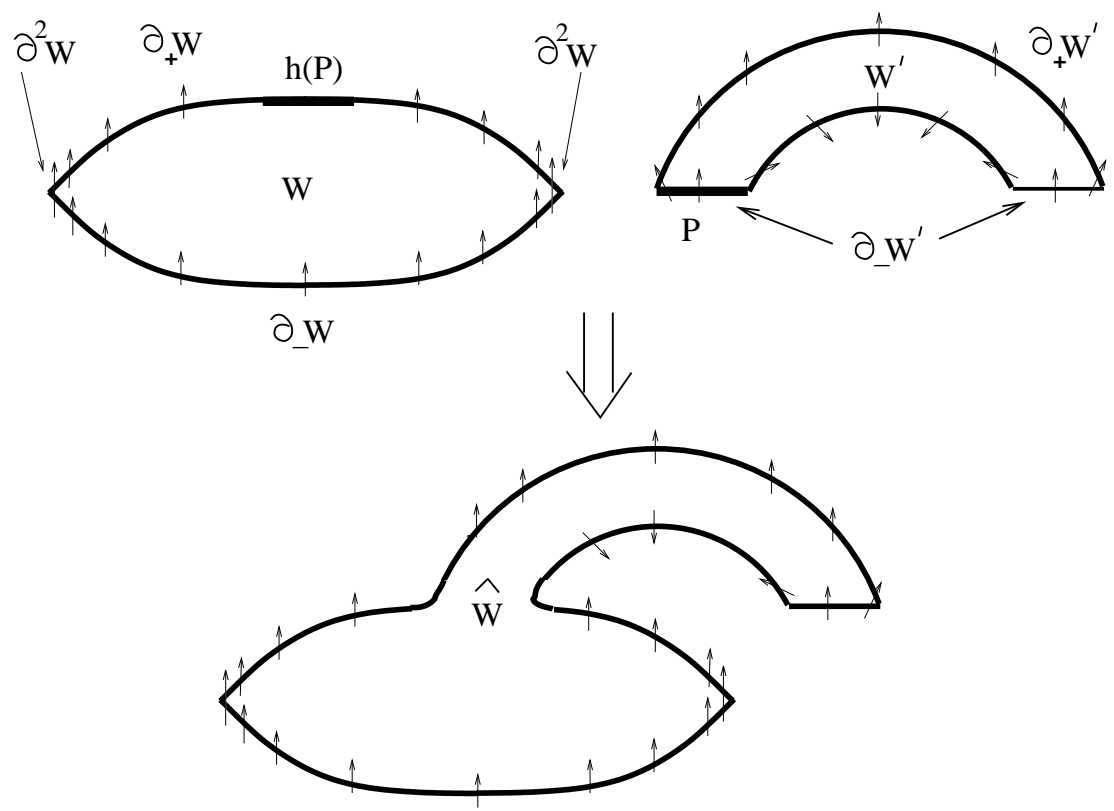

Fig. 2.4: concatenation of sutured cobordisms

component of $\partial_{-} W^{\prime}$. Given an embedding $h: P \rightarrow \operatorname{Int} \partial_{+} W$ one can glue $W^{\prime}$ to $W$ along $P$, forming a manifold with boundary with corners $\widehat{W}:=W \underset{h}{\cup} W^{\prime}$. The smooth structure is determined by matching the vector fields $Z$ and $Z^{\prime}$. The resulting manifold $\widehat{W}$ can be viewed as a sutured cobordism

$$
\left(\widehat{W}, \partial_{-} \widehat{W}:=\partial_{-} W \cup\left(\partial_{-} W^{\prime} \backslash P\right), \partial_{+} \widehat{W}:=\left(\partial_{+} W \backslash \operatorname{Int} P\right) \cup \partial_{+} W^{\prime}\right)
$$

(after smoothing all non-essential corners along $\partial P$ ). We say that $\widehat{W}$ is a result of concatenation of $W$ and $W^{\prime}$, see Figure 2.4. We will sometimes consider also an operation of backward concatenation, see Figure 2.5. For that we choose a connected component $P$ of $\partial_{+} W^{\prime}$ and an embedding $h: P \rightarrow \operatorname{Int} \partial_{-} W$, then we glue $W^{\prime}$ to $W$ along $P$, forming $\widehat{W}:=W^{\prime} \bigcup_{h} W$, while also matching the vector fields $Z^{\prime}$ and $Z$. The resulting manifold $\widehat{W}$ can be viewed as a sutured cobordism

$$
\left(\widehat{W}, \partial_{+} \widehat{W}:=\left(\partial_{+} W^{\prime} \backslash P\right) \cup \partial_{+} W, \partial_{-} \widehat{W}:=\left(\partial_{-} W \backslash \operatorname{Int} P\right) \cup \partial_{-} W^{\prime}\right),
$$

after smoothing all non-essential corner along $\partial P$.

A special case of the concatenation operation is handle attachment. In that case $W^{\prime}$ is the handle $D^{k} \times D^{n-k}$ with $\partial_{-} W^{\prime}=\partial D^{k} \times D^{n-k}, \partial_{+} W^{\prime}=D^{k} \times \partial D^{n-k}$ and the 


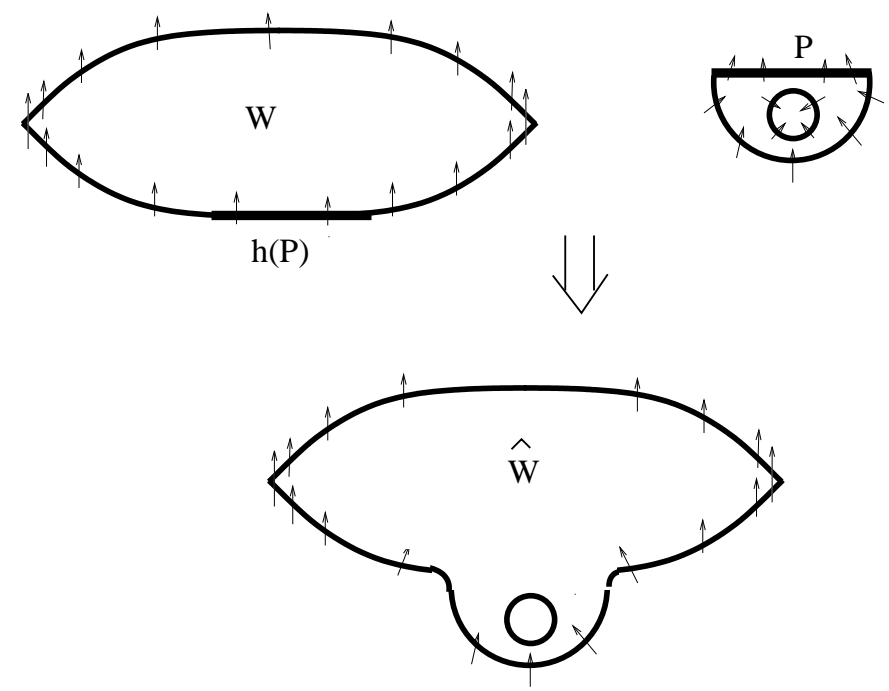

Fig. 2.5: Backward concatenation

and $P=\partial_{-} W^{\prime}$. To attach a handle to another sutured cobordism $\mathrm{W}$, we require an embedding $P=\partial D^{k} \times D^{n-k} \rightarrow$ Int $\partial_{+} W$, and then apply the concatenation operation described above. ${ }^{2}$

The next operation we will consider is another important instance of the concatenation operation.

Boundary inversion. Let $\left(W ; \partial_{-} W, \partial_{+} W\right)$ be a sutured cobordism, and let $U \subset$ Int $\partial_{+} W$ be a compact domain with smooth boundary. Let us recall that the definition of a sutured cobordism $W$ involves a vector field $Z$ on $\mathcal{O} p \partial W$ which is inwardly transverse to $\partial_{-} W$ and outwardly transverse to $\partial_{+} W$. Let us modify $\partial_{+} W$ by creating a corner along $\partial U$ and changing $Z$ on $\mathcal{O} p U$ in such a way that the new vector field $\hat{Z}$ is inward transverse to $U$ and outward transverse to $\partial_{+} W \backslash \operatorname{Int} U$. We say that the resulting cobordism

$$
\left(\widetilde{W}=W, \partial_{-} \widetilde{W}:=\partial_{-} W \cup U, \partial_{+} \widetilde{W}=\partial_{+} W \backslash \operatorname{Int} U ; \widetilde{Z}\right)
$$

is obtained from $\left(W ; \partial_{-} W, \partial_{+} W\right)$ by inverting the domain $U \subset \partial_{+} W$.

The inversion operation can be equivalently described as concatenation as follows. Take $W^{\prime}:=U \times[0,1]$ and view it as a sutured cobordism $\left(W^{\prime}, \partial_{-} W=(U \times 0 \cup\right.$ $\left.U \times 1), \partial_{+} W:=\partial U \times[0.1]\right)$. Let $\widehat{W}$ be the result of concatenating $W$ and $W^{\prime}$ by

\footnotetext{
${ }^{2}$ Throughout the paper we use the letters $D$ and $B$ to denote closed and open discs, respectively.
} 

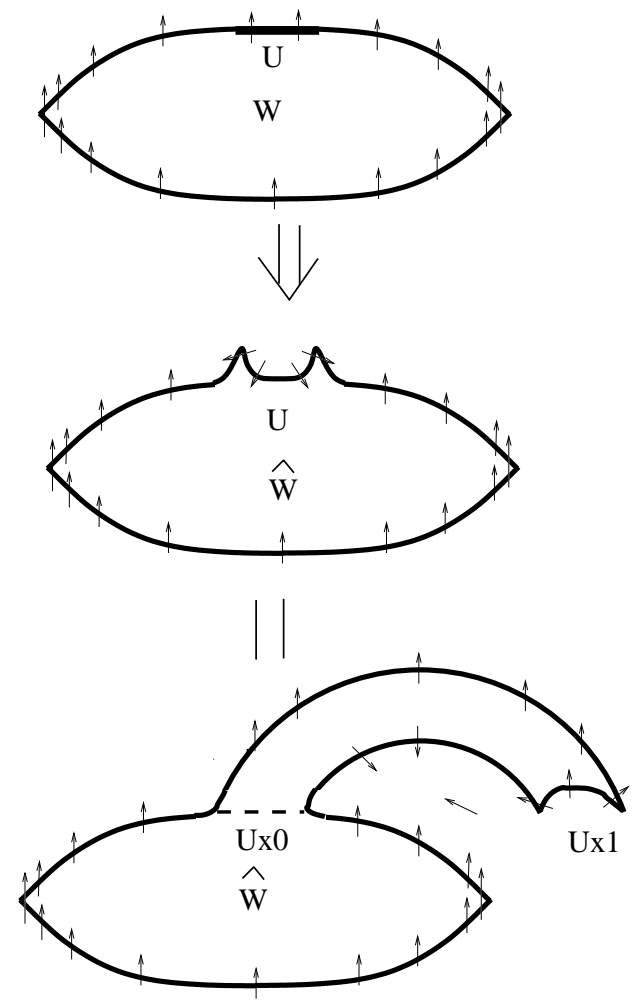

Fig. 2.6: Inverting the domain $U \subset \partial_{+} W$

identifying the component $U \times 0 \subset \partial_{-} W^{\prime}$ with $U \subset \operatorname{Int} \partial_{+} W$. The cobordism $\widehat{W}$, after smoothing non-essential corners, is diffeomorphic to $\widetilde{W}$, see Figure 2.6 .

Suture gluing. Let $W, W^{\prime}$ be sutured cobordisms with sutures $S=\partial^{2} W, S^{\prime}=\partial^{2} W^{\prime}$. Consider domains $P \subset S, P^{\prime} \subset S^{\prime}$ with smooth boundaries. Given a diffeomorphism $g: P \rightarrow P^{\prime}$ one can glue the cobordisms $W$ and $W^{\prime}$ to get a sutured cobordism $\widehat{W}$ with $\partial_{+} \widehat{W}=\partial_{+} W \underset{g}{\cup} \partial_{+} W^{\prime}, \partial_{-} \widehat{W}=\partial_{-} W \underset{g}{\cup} \partial_{-} W^{\prime}$. The new suture $\widehat{S}$ is equal to $\overline{S \backslash P} \cup \overline{S^{\prime} \backslash P^{\prime}}$ which is obtained by shaving parts $P, P^{\prime}$ of the sutures and gluing the resulted boundary components $P \times I$ and $P^{\prime} \times I$. See Figure 2.7 .

A Morse function $\phi: W \rightarrow \mathbb{R}$ on a sutured cobordism $(W, Z)$ is called admissible if the vector field $Z$ is gradient-like for $\left.\phi\right|_{\mathcal{O} p \partial W}$. We stress the point that even when $\partial^{2} W=\varnothing$, we do not require $\phi$ to be constant on $\partial_{ \pm} W$ (in contrast with the usual set-up of Morse theory). If $\partial^{2} W=\varnothing$ one can always modify $\phi$ on $\mathcal{O} p \partial W$ without 

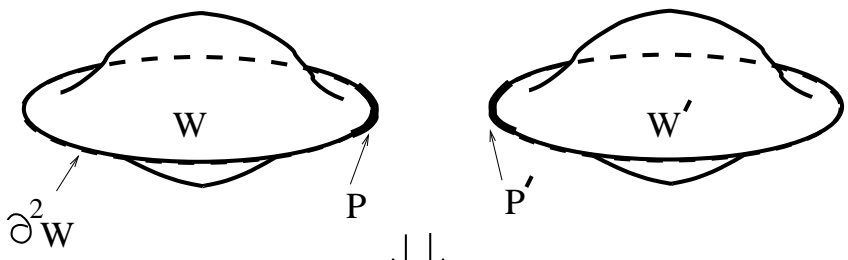

$\downarrow$
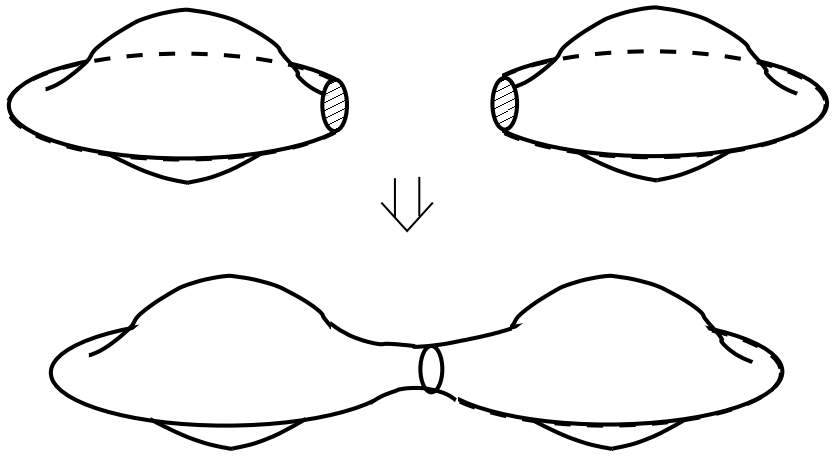

Fig. 2.7: Suture gluing.

creating additional critical points to make it constant on the boundary components.

Definition. Given a (sutured) cobordism $\left(W, \partial_{-} W, \partial_{+} W\right)$ we define the relative Morse type $\operatorname{Morse}\left(W, \partial_{-} W\right)$ of $\left(W, \partial_{-} W\right)$ as the minimal integer $m$ such that $W$ admits an admissible Morse function $\phi: W \rightarrow \mathbb{R}$ whose critical points have index $\leqslant m$. If $\partial_{-} W=\varnothing$ we will write $\operatorname{Morse}(W)$ instead of $\operatorname{Morse}(W, \varnothing)$.

Note that the pair $\left(W, \partial_{+} W\right)$ is $k$-connected, where $k=\operatorname{dim} W-\operatorname{Morse}\left(W, \partial_{-} W\right)-1$.

Lemma 2.2. (i) Let $\widehat{W}$ be the result of concatenation or suture gluing of sutured cobordisms $W$ and $W^{\prime}$. Then

$$
\operatorname{Morse}\left(\widehat{W}, \partial_{-} \widehat{W}\right) \leqslant \max \left(\operatorname{Morse}\left(W, \partial_{-} W\right), \operatorname{Morse}\left(W^{\prime}, \partial_{-} W^{\prime}\right)\right) \text {. }
$$

(ii) Suppose that the cobordism $\left(\widehat{W}, \partial_{-} \widehat{W}, \partial_{+} \widehat{W}\right)$ is obtained from $\left(W ; \partial_{-} W, \partial_{+} W\right)$ by inverting the domain $U \subset \partial_{+} W$. Then

$$
\operatorname{Morse}\left(\widehat{W}, \partial_{-} \widehat{W}\right) \leqslant \max \left(\operatorname{Morse}\left(W, \partial_{-} W\right), \operatorname{Morse}(U)+1\right)
$$

Proof. The proof of statement (i) is straightforward. Statement (ii) follows from (i) and the description of the boundary inversion as a concatenation of $W$ and $U \times I$, taking into account that $\operatorname{Morse}(U \times I, U \times \partial I) \leqslant \operatorname{Morse}(U)+1$. 

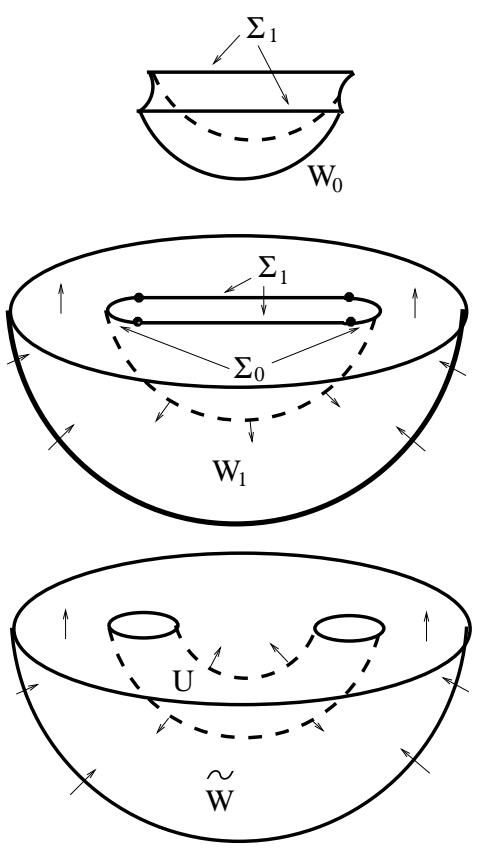

Fig. 2.8: Building cobordism $\widetilde{W}$

Lemma 2.3. Let $W$ be an $m$-dimensional sutured cobordism and $N$ a $k$-dimensional, $k<m-1$, manifold with boundary. Let $\hat{N}$ be the sutured version of the trivial cobordism $N \times[-1,1]$, see Remark 2.1a). Suppose we are given an embedding $\phi$ : $\left(\widehat{N}, \partial_{+} \hat{N}=N\right) \rightarrow\left(W\right.$, Int $\left.\partial_{+} W\right)$ which is transverse to $\partial_{+} W$. Let $U$ be a tubular neighborhood of $\partial_{-} \widehat{N}$ in $W$. Consider $\widetilde{W}:=\overline{W \backslash U}$, viewed as a sutured cobordism with $\partial_{-} \widetilde{W}=\partial_{-} W \cup\left(\Sigma:=\overline{\partial U \backslash \partial_{+} W}\right)$ and $\partial_{+} \widetilde{W}:=\overline{\partial_{+} W \backslash U}$. Then

$$
\operatorname{Morse}\left(\widetilde{W}, \partial_{-} \widetilde{W}\right) \leqslant \max \left(\operatorname{Morse}\left(W, \partial_{-} W\right), \operatorname{Morse}(N)+1\right) \text {. }
$$

Proof. The cobordism $\left(\widetilde{W}, \partial_{-} \widetilde{W}, \partial_{+} \widetilde{W}\right)$ can be equivalently described as follows. Choose any Riemannian metric on $\partial_{+} W$ and for a sufficiently small $\varepsilon>0$ consider an $\varepsilon$-neighborhood $V$ of $\phi(N)=\phi\left(\partial_{+} \widehat{N}\right)$ in $\partial_{+} W$. The neighborhood $V$ is the union of

(i) the total space of a bundle over $\phi(\partial N)$ whose fibers are $(m-k+1)$-dimensional half-discs of radius $\varepsilon$ and

(ii) the total space of the normal $\varepsilon$-disc bundle of rank $m-k$ over $\phi(N)$. 
We denote the total spaces of the associated half-sphere and sphere bundles by $\Sigma_{0}$ and $\Sigma_{1}$, respectively. We have $\partial V=\Sigma_{0} \cup \Sigma_{1}$. Consider the sutured cobordism $W_{1}$ obtained from $W$ by inverting the domain $V$ in $\partial_{+} W$, and let $W_{0}$ be the sutured version of the trivial cobordism $V \times[-1,1]$. Note that $\partial V$ is one of the suture components of $W_{1}$, and at the same time it is the sole suture of $W_{0}$. Performing the suture gluing operation of $W_{1}$ and $W_{0}$ along the domain $\Sigma_{1} \subset \partial V$ we obtain a sutured cobordism isomorphic to $\widetilde{W}$, see Figure 2.8. Because $W_{0}$ is a trivial cobordism, $\operatorname{Morse}\left(W_{0}, \partial_{-} W_{0}\right)=0$, while according to Lemma 2.2(ii) we have

$$
\operatorname{Morse}\left(W_{1}, \partial_{-} W_{1}\right) \leqslant \max \left(\operatorname{Morse}\left(W, \partial_{-} W\right), \operatorname{Morse}(V)+1\right) .
$$

Hence, using Lemma 2.2(i) we conclude

$$
\begin{aligned}
& \operatorname{Morse}(\widetilde{W}, \partial \widetilde{W}) \leqslant \max \left(\operatorname{Morse}\left(W_{1}, \partial W_{1}\right), \operatorname{Morse}\left(W_{0}, \partial W_{0}\right)\right) \\
& \leqslant \max \left(\operatorname{Morse}\left(W, \partial_{-} W\right), \operatorname{Morse}(V)+1\right)=\max \left(\operatorname{Morse}\left(W, \partial_{-} W\right), \operatorname{Morse}(N)+1\right),
\end{aligned}
$$

as desired.

Lemma 2.4. Given positive integers $m, k, \ell>0$ such that $m>k+\ell$ consider an index $m-k$ handle $H=D^{m-k} \times D^{k}$, viewed as a sutured cobordism with $\partial_{-} H=$ $\partial D^{m-k} \times D^{k}$ and $\partial_{+} H=D^{m-k} \times \partial D^{k}$. Let $D^{\ell} \subset D^{m-k}$ be the equatorial disk cut out by the first $\ell$ coordinates. Denote $S:=\partial D^{\ell}\left(\frac{1}{2}\right) \times D^{k}$, and let $V$ be a tubular neighborhood of $S$ in $H$. Here the notation $D^{j}(r)$ stands for a $j$-dimensional disc of radius $r$ and we write $D^{j}$ for $D^{j}(1)$. Consider the sutured cobordism

$$
\left(W:=\overline{H \backslash V}, \partial_{+} W:=\overline{\partial_{+} H \backslash V \cup \partial V \backslash \partial_{+} H}, \partial_{-} W:=\partial_{-} H\right) .
$$

Then

$$
\operatorname{Morse}\left(W, \partial_{-} W\right)=m-k-\ell \text {. }
$$

Proof. Let us give a different equivalent definition of the cobordism $W$. Denote

$$
\Sigma:=D^{\ell}\left(\frac{1}{2}\right) \times D^{k}, \breve{V}:=\Sigma \times D^{m-k-\ell}(\varepsilon) .
$$

After smoothing non-essential corners we can view $\breve{V}$ as a sutured cobordism with $\partial_{-} \breve{V}:=D^{\ell}\left(\frac{1}{2}\right) \times D^{k} \times \partial D^{m-k-\ell}(\varepsilon)$ and $\partial_{+} \breve{V}:=\partial\left(D^{\ell}\left(\frac{1}{2}\right) \times D^{k}\right) \times D^{m-k-\ell}(\varepsilon)$. 

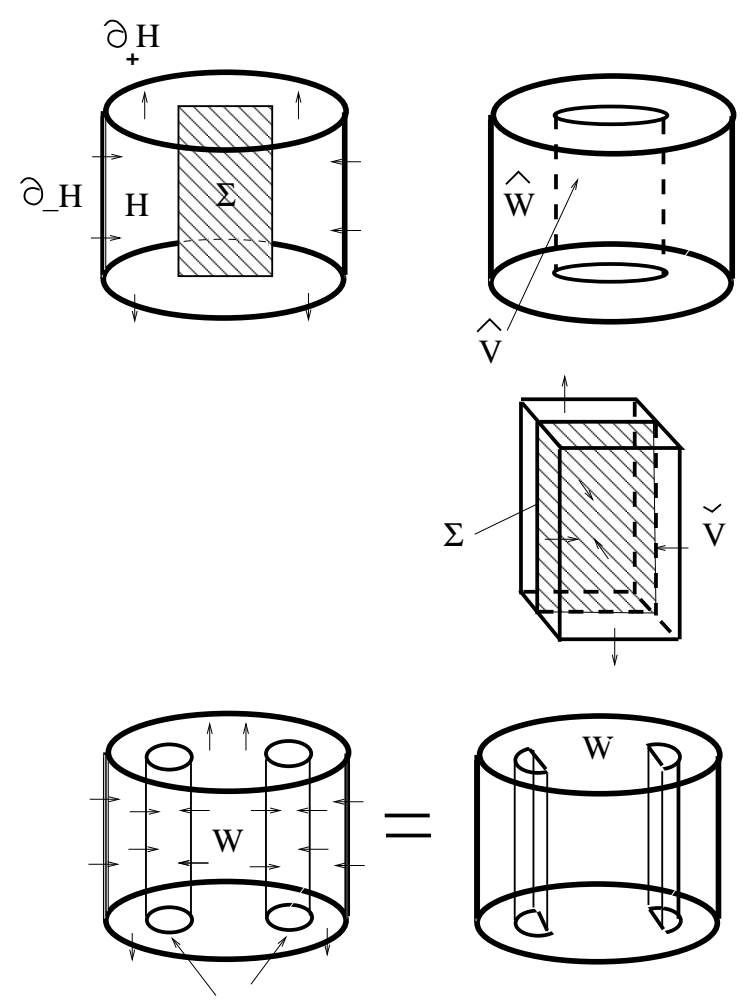

$\mathrm{U}$

Fig. 2.9: Two constructions of $W ; m=3, k=\ell=1$.

Let $\widehat{V}$ be the $\varepsilon$-neighborhood of the submanifold $\Sigma \subset H$. Consider a cobordism $\widehat{W}$ obtained from $H$ by removing $\widehat{V}$ :

$$
\left.\widehat{W}=\overline{H \backslash \widehat{V}}, \partial_{+} \widehat{W}=\overline{\partial_{+} H \backslash \widehat{V}} \cup \overline{\partial \widehat{V} \backslash \partial_{+} H}, \partial_{-} \widehat{W}=\partial_{-} H\right) .
$$

Note that $\partial_{-} \breve{V}$ is contained in $\partial_{+} \widehat{W}$ and by concatenating $\widehat{W}$ and $\breve{V}$ and then smoothing non-essential corners we get a sutured cobordism diffeomorphic to $W$, see Fig. 2.9. But $\widehat{V}$ is equivalent to a sutured version of the trivial cobordism over $\partial_{-} H$, while $V$ is just an index $m-k-\ell$ handle. Hence, $\operatorname{Morse}\left(W, \partial_{-} W\right)=m-k-\ell$.

\subsection{Liouville and Weinstein sutured cobordisms}

A sutured cobordism $(W, Z)$ is called Liouville if it is endowed with an exact symplectic form $\omega=d \lambda$, and the vector field $Z$ is the Liouville vector field dual to the 
Liouville form $\lambda$.

Any (sutured) Liouville cobordism can always be completed by attaching to $\partial_{+} W$ the positive part of the symplectization $\left(\partial_{+} W \times[0, \infty), e^{s}\left(\left.\lambda\right|_{\partial_{+} W}\right)\right)$, and to $\partial_{-} W$ the negative part of the symplectization $\left(\partial_{+} W \times(-\infty, 0], e^{s}\left(\left.\lambda\right|_{\partial_{-} W}\right)\right)$, and matching the Liouville field $\left.Z\right|_{\partial_{ \pm} W}$ with the vertical vector field $\frac{\partial}{\partial s}$, see Fig. 2.10. The resulting exact symplectic manifold (potentially with boundary) is denoted $\widetilde{W}$, and is called the completion of $W$.

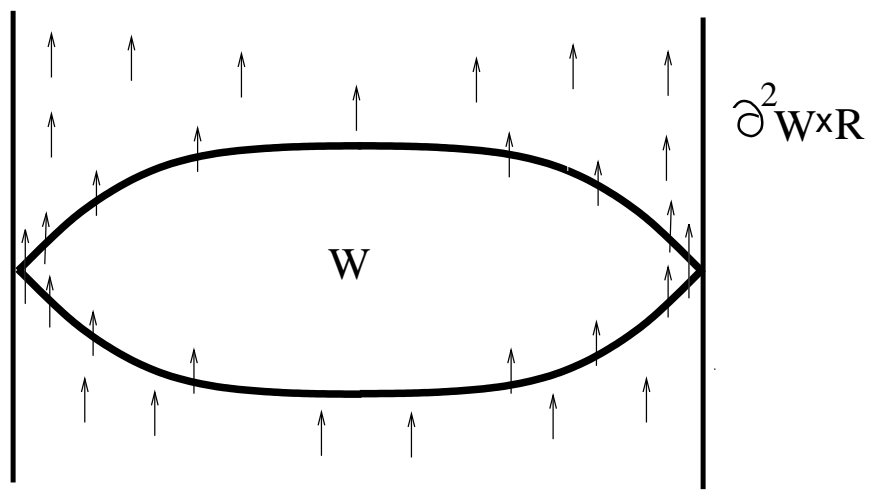

Fig. 2.10: Attaching cylindrical ends.

By construction, the Liouville vector field $Z$ extends from $W$ to a complete Liouville field, still denoted by $Z$ on the completion $\widehat{W} \supset W$. In the case when $\partial^{2} W \neq \varnothing$ the manifold $\widehat{W}$ has a boundary $\partial^{2} W \times \mathbb{R}$ where each fiber $x \times \mathbb{R}$ is the complete $Z$-trajectory through $x \in \partial^{2} W$. The completion construction yields the following useful statement.

Lemma 2.5. Let $(W, \lambda)$ be a sutured Liouville cobordism. Denote $\alpha_{ \pm}:=\left.\lambda\right|_{\partial_{ \pm} W}$. Then for any positive function $h_{+}: \partial_{+} W \rightarrow \mathbb{R}$ there exists a Liouville cobordism structure $\tilde{\lambda}$ on $W$ and a function $h_{-}: \partial_{-} W \rightarrow \mathbb{R}$ which can be chosen to agree with $h_{+}$on $\mathcal{O} p \partial^{2} W$, such that $\left.\widetilde{\lambda}\right|_{\partial_{+} W}=h_{ \pm} \alpha_{ \pm}$. Similarly, for any positive function $h_{-}: \partial_{-} W \rightarrow \mathbb{R}$ there exists a Liouville cobordism structure $\tilde{\lambda}$ on $W$ and a function $h_{+}: \partial_{+} W \rightarrow \mathbb{R}$ which can be chosen equal to $h_{-}$on $\mathcal{O} p \partial^{2} W$ such that $\left.\tilde{\lambda}\right|_{\partial_{ \pm} W}=h_{ \pm} \alpha_{ \pm}$. If the function $h_{+}$(resp. $h_{-}$) is $\geqslant 1$ (resp. $\leqslant 1$ ) and $=1$ on $\partial^{2} W$ then the function $h_{-}\left(\right.$resp. $\left.h_{+}\right)$can be chosen $\equiv 1$.

Proof. Consider the completion $\widehat{W} \supset W$. For any smooth function $g: \partial_{ \pm} W \rightarrow \mathbb{R}$ 
denote by $\phi_{ \pm}^{g}$ the embedding $\partial_{ \pm} W \rightarrow \widehat{W}$ given by the formula

$$
\phi_{ \pm}^{g}(x)=Z^{g(x)}(x), \quad x \in \partial_{ \pm} W
$$

where $Z^{t}: \widehat{W} \rightarrow \widehat{W}$ is the time $t$ flow of the vector field $Z$. Note that $\left(\phi_{ \pm}^{g}\right)^{*} \lambda=e^{g} \alpha_{ \pm}$. Denote $\Sigma_{ \pm}^{g}:=\phi_{ \pm}^{g}\left(\partial_{ \pm} W\right)$.

Let $g_{+}:=\ln h_{+}$, and choose a function $g_{-}$agreeing with $g_{+}$on $\partial^{2} W$, and so that the two hypersurfaces $\Sigma_{+}^{g_{+}}$and $\Sigma_{-}^{g_{-}}$bound in $\widehat{W}$ a sutured cobordism $W^{g_{+}, g_{-}}$(the second condition imposes a restriction on how large $g_{-}$can be). If these two conditions are satisfied, the embeddings $\phi_{+}^{g_{+}}$and $\phi_{-}^{g_{-}}$extend to a diffeomorphism $G: W \rightarrow W^{g_{+}, g_{-}}$. Then the Liouville form $G^{*} \lambda$ restricts to $\partial_{+} W$ as $e^{g_{+}} \alpha_{+}$and to $\partial^{-} W$ as $e^{g_{-}} \alpha_{-}$, i.e. $h_{-}:=e^{g_{-}}$is the required function. Similarly, setting $g_{-}:=\ln h_{-}$we can find a function $g_{+}$equal to $g_{-}$on $\partial^{2} W$ such that the above claims hold, i.e. $\Sigma_{+}^{g_{+}}$and $\Sigma_{-}^{g_{-}}$ bound in $\widehat{W}$ a sutured cobordism $W^{g_{+}, g_{-}}$; then $h_{+}:=e^{g_{+}}$is the required function. If $g_{+} \geqslant 0$ and $\left.g_{+}\right|_{\partial^{2} W}=0$ then one can choose $g_{-} \equiv 0$, and similarly if $g_{-} \leqslant 0$ and $\left.g_{-}\right|_{\partial^{2} W}=0$ then one can choose $g_{+} \equiv 0$.

A (sutured) Liouville cobordism $(W, \omega=d \lambda)$ is called Weinstein if the corresponding Liouville vector field $Z$ is gradient-like for a Morse function $\phi: W \rightarrow \mathbb{R}$. In the Weinstein case the critical points of the function $\phi$ have index $\leqslant n=\frac{1}{2} \operatorname{dim} W$, see [9], and hence $\operatorname{Morse}\left(W, \partial_{-} W\right) \leqslant n$.

Let us now consider the Liouville and Weinstein versions of the operations on sutured cobordisms introduced above in Section 2.1.

To define the concatenation of two Liouville cobordisms $(W, \lambda),\left(W^{\prime}, \lambda^{\prime}\right)$ choose a component $P$ of $\partial_{-} W^{\prime}$ and a contact form preserving embedding

$$
h:\left(P,\left.\lambda^{\prime}\right|_{P}\right), \rightarrow\left(\operatorname{Int} \partial_{+} W,\left.\lambda\right|_{\partial_{+} W}\right) .
$$

This allows us to match the corresponding Liouville forms (as two Liouville forms on $\mathcal{O} p \partial_{-} W^{\prime}$ which coincide on $\partial_{-} W^{\prime}$ are isotopic) and hence define a Liouville cobordism structure on the result of the concatenation operation. Corner smoothing at nonessential corners, as defined above, uses the Liouville vector field $Z$, and hence will keep the boundary faces contact. Similarly, one defines the suture gluing operation of two sutured Liouville cobordisms if the matching diffeomorphism $g: U \rightarrow U^{\prime}$ is chosen to preserve the induced forms: $g^{*}\left(\left.\lambda\right|_{U} ^{\prime}\right)=\left.\lambda\right|_{U}$.

The boundary inversion operation is more delicate in the Liouville context. It can be performed only for a restricted class of domains $U \subset \partial_{+} W$, e.g. neighborhoods of 
Legendrian submanifolds, or, more generally, neighborhoods of Liouville hypersurfaces, see [7].

The concatenation, gluing and inversion operations can also be performed in the category of sutured Weinstein cobordisms.

Remark 2.6. When considering sutured Liouville and Weinstein cobordisms, or equivalently Liouville and Weinstein cobordisms between contact manifolds with boundary, it is customary to impose some constrains on the boundaries of the contact manifolds, e.g. contact convexity, in order to enable the application of methods from the theory of holomorphic curves. However, the main results of this paper hold without any such constraints.

\subsection{Attaching and subtracting Weinstein handles}

Let us recall some basic definitions and statements from the Weinstein surgery theory. For more detail, see [3, 5, 27].

Let $\left(\mathbb{R}^{2 n}, \sum_{j=1}^{n} d p_{j} \wedge d q_{j}\right)$ be the standard symplectic space. A Weinstein handle $H_{k}$ of index $k \leqslant n$ is the domain

$$
H_{k}=\left\{\sum_{i=1}^{k} q_{i}^{2} \leqslant 1, \sum_{i=k+1}^{n} q_{i}^{2}+\sum_{i=1}^{n} p_{i}^{2} \leqslant 1\right\} \subset \mathbb{R}^{2 n}
$$

endowed with the Liouville form

$$
\lambda_{k}=\sum_{i=1}^{k}\left(2 p_{i} d q_{i}+q_{i} d p_{i}\right)+\sum_{i=k+1}^{n} p_{i} d q_{i} .
$$

We have $d \lambda_{k}=\sum_{1}^{n} d p_{i} \wedge d q_{i}$.

The isotropic $k$-disc

$$
D:=\left\{q_{k+1}=\cdots=q_{n}=0, p_{1}=\cdots=p_{n}=0\right\} \subset H_{k}
$$

is called the core disc of the handle and the coisotropic $(2 n-k)$-disc

$$
C:=\left\{q_{1}=\cdots=q_{k}=0\right\} \subset H_{k}
$$

the co-core of the handle. We have a canonical decomposition $H_{k}=D \times C$. Denote $\Lambda_{-}:=\partial D, \Lambda_{+}:=\partial C$. The handle $H_{k}$ can be viewed as a Weinstein sutured 
cobordism with

$$
\begin{aligned}
& \partial_{-} H_{k}:=\Lambda_{-} \times C=\left\{\sum_{i=1}^{k} q_{i}^{2}=1\right\} \cap H_{k}, \\
& \partial_{+} H_{k}:=\Lambda_{+} \times D=\left\{\sum_{i=k+1}^{n} q_{i}^{2}+\sum_{i=1}^{n} p_{i}^{2}=1\right\} \cap H_{k} .
\end{aligned}
$$

Indeed, the Liouville vector field

$$
Z_{k}=\sum_{i=1}^{k}\left(2 p_{i} \frac{\partial}{\partial p_{i}}-q_{i} \frac{\partial}{\partial q_{i}}\right)+\sum_{i=k+1}^{n} p_{i} \frac{\partial}{\partial p_{i}}
$$

is inwardly transverse to $\partial_{-} H_{k}$ and outwardly transverse to $\partial_{+} H_{k}$. The function $\sum_{k+1}^{n} q_{j}^{2}+\sum_{1}^{n} p_{i}^{2}-\sum_{1}^{k} q_{j}^{2}$ serves as a Lyapunov function for the Liouville vector field $Z_{k}$. We will denote by $\xi_{k, \pm}$ the contact structure on $\partial_{ \pm} H^{k}$ defined by the contact form $\lambda_{k, \pm}:=\left.\lambda_{k}\right|_{\partial_{ \pm} H_{k}}$.

Let $(W, \lambda)$ be a (sutured) Liouville cobordism, and suppose $h_{-}:\left(\partial_{-} H_{k}, \lambda_{k,-}\right) \rightarrow$ $\left(\partial_{+} W,\left.\lambda\right|_{\partial_{+} W}\right)$ is a contact form preserving embedding. Then we can attach the handle $H_{k}$ to $W$ using $h_{-}$by concatenating $W$ and $H_{k}$, as described in the previous section. We say that the resulting sutured Liouville cobordism $(\widetilde{W}, \tilde{\lambda}) \supset(W, \lambda)$ is obtained from $W$ by attaching an index $k$ Weinstein handle. Similarly, given a contact form preserving embedding $h_{+}:\left(H_{+}^{k}, \lambda_{k,+}\right) \rightarrow\left(\partial_{-} W,\left.\lambda\right|_{\partial_{-} W}\right)$, we may consider the backward concatenation of $H_{k}$ and $W$.

The effect of a Weinstein handle attachment to the positive contact boundary $\partial_{+} W$ is called the direct Weinstein surgery of index $k$, while the effect of a backward attachment to the negative contact boundary $\partial_{-} W$ is called the inverse Weinstein surgery of index $2 n-k 3^{3}$

On the other hand, if we are given a Liouville embedding $G:\left(H_{k}, \partial_{-} H_{k} ; \lambda_{k}\right) \rightarrow$ $\left(W, \partial_{-} W ; \lambda\right)$ (resp. $\left.G:\left(H_{k}, \partial_{+} H_{k} ; \lambda_{k}\right) \rightarrow\left(W, \partial_{+} W ; \lambda\right)\right)$ of the whole handle, then one can subtract the handle, thus obtaining a new Liouville cobordism

$$
\left(\widetilde{W}:=\overline{W \backslash G\left(H_{k}\right)}, \widetilde{\lambda}=\left.\lambda\right|_{\widetilde{W}}\right) \subset(W, \lambda)
$$

such that

$$
\begin{aligned}
& \partial_{+} \widetilde{W}:=\partial_{+} W \text { and } \partial_{-} \widetilde{W}:=\overline{\partial_{-} W \backslash G\left(\partial_{-} H_{k}\right)} \cup G\left(\partial_{+} H_{k}\right), \\
& \left(\operatorname{resp} . \partial_{-} \widetilde{W}:=\partial_{-} W \text { and } \partial_{+} \widetilde{W}:=\overline{\partial_{+} W \backslash G\left(\partial_{+} H_{k}\right)} \cup G\left(\partial_{-} H_{k}\right) .\right)
\end{aligned}
$$

\footnotetext{
3 The direct and inverse Weinstein surgeries are also called (-1)- or (+1)-surgeries, respectively.
} 
In fact, the result of this operation is determined (up to isomorphism) by the isotropic embedding $\left.G\right|_{D}$, where $D \subset H_{k}$ is the core disc (resp. the co-isotropic embedding $\left.G\right|_{C}$ where $C \subset H_{k}$ is the co-core disc).

The following lemma is a corollary of the classification of overtwisted contact structures established in [1, see Theorem 1.4 in the current paper.

Lemma 2.7. Let $(Y, \xi)$ be an overtwisted contact manifold. Then any almost contact embedding $h_{ \pm}:\left(\partial_{ \pm} H_{k}, \xi_{k, \pm}\right) \rightarrow(Y, \xi)$ is isotopic to a genuine contact embedding. We can furthermore ensure that the complement of the image $h_{ \pm}\left(\partial_{ \pm} H_{k}\right)$ is overtwisted.

We recall that given two contact manifolds $(M, \xi)$ and $(N, \eta)$, an almost contact embedding is a pair $\left(f, \Phi_{t}\right)$ where $f: M \rightarrow N$ is a smooth embedding and $\Phi_{t}$ : $T M \rightarrow T N$ is a homotopy of injective homomorphisms covering $f$ such that $\Phi_{0}=d f$, $\Phi_{1}(\xi) \subset \eta$ and $\left.\Phi_{1}\right|_{\xi}: \xi \rightarrow \eta$ is a fiberwise conformal symplectic homomorphism with respect to the symplectic structures induced on $\xi$ and $\eta$ by any choice of contact forms. A genuine contact embedding $f: M \rightarrow N$ can be viewed as almost contact by adding a homotopy $\Phi_{t}:=d f$.

Proof of Lemma 2.7. We will prove below a more general statement: any almost contact embedding $\left(f, \Phi_{t}\right):(M, \xi) \rightarrow(N, \eta)$ between two contact manifolds, where $\eta$ is overtwisted, is isotopic through the space of almost contact embeddings to a genuine contact embedding with an overtwisted complement. Note that the normal bundle $\nu$ to $\Phi_{1}(T M)$ in $T N$ has a symplectic structure $\bmod C^{\infty}(M, \mathbb{R})$, because $\nu$ is isomorphic to the quotient $\nu=\eta / \Phi_{1}(\xi)$ of two symplectic bundles $\bmod C^{\infty}(M, \mathbb{R})$. The homotopy $\Phi_{t}$ allows us to pull back this structure to the normal bundle $\widetilde{\nu}$ to $f(M)$ in $N$. A neighborhood $U$ of the 0 -section $M$ in the total space $E$ of the bundle $f^{*} \widetilde{\nu}$ over $M$ has a canonical, up to fixed on $M$ isotopy, contact structure $\tilde{\xi}$ by Darboux (-Givental)'s theorem. Having chosen such a neighborhood $U$, let us extend $f$ to a diffeomorphism $F$ of $U$ onto a tubular neighborhood of $f(M)$ in $N$. We can extend the homotopy $\Phi_{t}$ to a homotopy $\widetilde{\Phi}_{t}$ of fiberwise isomorphisms $T U \rightarrow T N$, covering $F$ and such that $\widetilde{\Phi}_{0}=d F, \widetilde{\Phi}_{1}(\widetilde{\xi})=\eta$ and $\left.\widetilde{\Phi}_{1}\right|_{\widetilde{\xi}}: \widetilde{\xi} \rightarrow \eta$ is a conformally symplectic isomorphism. The push-forward $\left(\widetilde{\Phi}_{t}\right)_{*} \xi$ is a homotopy of almost contact structures on $F(U)$ connecting $F_{*} \widetilde{\xi}$ with $\eta$. This homotopy can be extended to a homotopy $\eta_{t}$ of almost contact structures on all of $N$ connecting $\eta_{1}=\eta$ with an almost contact structure $\eta_{0}$ which coincides with the genuine contact structure $F_{*} \widetilde{\xi}$ on $F(U)$. Hence, by applying the classification of overtwisted contact structures, we get a genuine overtwisted contact structure $\widetilde{\eta}$ on $N$ extending $F_{*} \widetilde{\xi}$, which is in the same homotopy class of almost contact structures as $\eta$. Therefore, according 
to Theorem $1.4 \widetilde{\eta}$ is also in the same homotopy class of genuine contact structures and moreover, we can ensure that the contact structure $\widetilde{\eta}$ is overtwisted on every component of the complement $N \backslash F(U)$. By Gray's theorem [15] there is a diffeotopy $\phi_{t}: N \rightarrow N$ such that $\phi_{0}=\operatorname{Id}$ and $\left(\phi_{1}\right)_{*} F_{*} \widetilde{\xi}=\eta$. Then $\phi_{1} \circ f$ is the required contact embedding $(M, \xi) \rightarrow(N, \eta)$.

The following theorem is a corollary of Lemma 2.7 and the results from [5] (see also [3]) which were proved using Weinstein handlebody constructions:

Theorem 2.8. Consider a $2 n$-dimensional connected almost symplectic sutured cobordism $(W, \eta)$. Let $\lambda$ be a Liouville form on $\mathcal{O}_{p} \partial_{-} W$ such that the corresponding Liouville field $Z$ is inward transverse to $\partial_{-} W$ and outward transverse to $\partial_{+} W \cap \mathcal{O}_{p} \partial_{-} W$. Suppose that the contact structure $\xi_{-}=\left\{\left.\lambda\right|_{\partial_{-} W}=0\right\}$ is compatible with $\eta$. If $n=2$ we assume that $\xi_{-}$is overtwisted on at least one of the components of $\partial_{-} W$. Suppose that $\operatorname{Morse}\left(W, \partial_{-} W\right) \leqslant n$. Then $\lambda$ extends to $W$ as a sutured Liouville cobordism structure with $\left(\partial_{+} W, \operatorname{Ker}\left(\left.\lambda\right|_{\partial_{+} W}\right)\right)$ and $\left(\partial_{-} W, \operatorname{Ker}\left(\left.\lambda\right|_{\partial_{+} W}\right)\right)$ as its positive and negative contact boundaries, and such that $d \lambda$ and $\eta$ are homotopic rel. $\partial_{-} W$ as almost symplectic forms. If $\xi_{-}$is overtwisted on at least one of the components of $\partial_{-} W$, then one can arrange that $\xi_{+}$is overtwisted as well (and in this case $\xi_{+}$is uniquely determined up to isotopy by the homotopy class of the almost symplectic structure $\eta)$.

Remark 2.9. 1. Connectedness of $W$ and the assumption Morse $\left(W, \partial_{-} W\right) \leqslant n$ imply that $\partial_{+} W$ is connected.

2. In fact, the construction in [5] and [3] yields a Weinstein (and not just Liouville) cobordism structure on $W$, but this result will not be needed for our purposes. Though the corresponding results are formulated in [5] and [3] for non-sutured cobordisms, the proof is local near the attaching spheres of the handles, and hence it works without any changes for the case when $\partial^{2} W \neq \varnothing$.

Theorem 2.8 together with Lemma 2.5 implies the following

Corollary 2.10. Consider a 2n-dimensional connected almost symplectic sutured cobordism $(W, \eta)$. Suppose that $\operatorname{Morse}\left(W, \partial_{-} W\right) \leqslant n$. Let $\lambda$ be a Liouville form on $\mathcal{O} p \partial W$ such that the corresponding Liouville field $Z$ is inwardly transverse to $\partial_{-} W$ and outwardly transverse to $\partial_{+} W$. Suppose that $\left.\eta\right|_{\mathcal{O}_{p} \partial W}=d \lambda$ and that both contact forms $\alpha_{ \pm}:=\left.\lambda\right|_{\partial_{ \pm} W}$ are overtwisted (if $\partial_{-} W$ is disconnected then we suppose that at least one of its components is overtwisted). Then there exists a Liouville form $\Lambda$ on $W$ such that 
(i) $d \Lambda$ is homotopic to $\eta$ via a homotopy of almost symplectic forms fixed on $\partial_{+} W$;

(ii) $\left.\Lambda\right|_{\partial_{+} W}=\alpha_{+}$;

(iii) $\left.\Lambda\right|_{\partial_{-} W}=h \alpha_{-}$for a function $h: \partial_{-} W \rightarrow(0,1]$ which is equal to 1 near $\partial\left(\partial_{-} W\right)=\partial^{2} W$.

The next proposition concerning an inverse Weinstein surgery on loose Legendrian knots is proven in [2] (see [24] for a definition of loose Legendrians). For the convenience of the reader we provide a modified proof here.

Proposition 2.11. The inverse surgery on a loose Legendrian knot produces an overtwisted contact manifold.

Proof. Let $\Lambda \subset(Y, \xi)$ be a loose Legendrian sphere. Note that for an arbitrary neighborhood of the 0 -section $U \subset T^{*} \Lambda$ the inclusion $\Lambda \hookrightarrow Y$ extends to a contact embedding $\left(U \times[-1,1], d z-\lambda_{\text {std }}\right) \rightarrow Y$. Present the sphere $\Lambda$ as a union $A \cup B:=$ $S^{1} \times D^{n-2} \cup D^{2} \times S^{n-3}$. Then $T^{*} \Lambda=T^{*} A \cup T^{*} B$ and $T^{*} A=T^{*} S^{1} \times T^{*} D^{n-2}$. Hence, we can choose the above neighborhood $U$ to contain the product of arbitrary large neighborhoods $U_{1}$ and $U_{2}$ of the 0 -sections in $T^{*} S^{1}$ and $T^{*} D^{n-2}$. We denote Liouville forms in $T^{*} S^{1}$ and $T^{*} D^{n-2}$ by $u d t$ and $\sum_{1}^{n-2} p_{i} d q_{i}$, respectively. Let $\Gamma_{\mathrm{st}}$ be a Legendrian stabilization of the 0 -section $\Gamma$ in the 3 -dimensional contact manifold $\left(V_{1}:=U_{1} \times[-1,1], d z-u d t\right)$. By attaching an inverse 4-dimensional handle $H_{2}$ along $\Gamma_{\text {st }}$ we get a symplectic cobordism $X$ with $\partial_{+} X=V_{1}$ and $\partial_{-} X=: V_{1}^{-}$. The negative boundary $\partial_{-} X$ is overtwisted, because a parallel copy of the zero section $\Gamma$ bounds a disk in $V_{1}^{-}$(after flowing from $\partial_{+} X$ to $\partial_{-} X$ ) and has 0 Thurston-Bennequin number. According to [24], there exists a Legendrian sphere $\Lambda_{\text {st }}$ in a neighborhood of $\Lambda$ which is Legendrian isotopic to $\Lambda$ in $(Y, \xi)$ and so that $\Lambda_{\text {st }} \cap V_{1} \times U_{2}=\Gamma_{\text {st }} \times\{p=0\}$. Then the Liouville manifold which we get by attaching the inverse handle $H_{n}$ along $\Lambda_{\text {st }}$ contains the product $X \times U_{2}$, and hence its negative contact boundary contains $V_{1}^{-} \times U_{2}$. But the neighborhood of the 0 -section $U_{2} \subset T^{*} D^{n-2}$ can be chosen arbitrarily large, and hence the resulting contact manifold is overtwisted by Theorem 1.5 .

Corollary 2.12 (see [2]). For any contact manifold $(Y, \xi)$ of dimension $>3$, there exists a Weinstein cobordism structure on $W=Y \times[0,1]$ between the contact structure $\xi$ on $\partial_{+} W:=Y \times 1$ and an overtwisted contact structure $\xi_{\text {ot }}$ on $\partial_{-} W:=Y \times 0$.

Proof. On the trivial Weinstein cobordism $W=Y \times[0,1]$ deform the Weinstein structure to create two critical points $a, b$ of index $n-1$ and $n$, respectively. Let 
$\left(Z, \xi_{Z}\right)$ be the intermediate level set for this cobordism. Let $\Gamma \subset Y \times 1$ be the unstable Legendrian sphere for the critical point $b$. Consider a stabilization $\widehat{\Gamma}$ of $\Gamma$ which is formally isotopic to $\Gamma$. By attaching an inverse handle $H_{n}$ to $(Y=Y \times 1, \xi)$ along $\widehat{\Gamma}$ we construct a Liouville cobordism between $Z$ and $Y$ with an overtwisted contact structure $\xi_{\text {ot }}$ on $Z$ in the formal class of $\xi_{Z}$. By Lemma 2.7 we can find a coisotropic embedding of an $(n+1)$-dimensional sphere into $\left(Z, \xi_{\text {ot }}\right)$ which is in the formal class of the unstable sphere of the critical point $a$ in $\left(Z, \xi_{Z}\right)$, and such that its complement is still overtwisted. Hence one can attach an inverse handle $H_{n-1}$ to $Z$ to get a Weinstein structure on the cobordism $Y \times[0,1]$ with the contact structure $\xi$ on the positive end and an overtwisted contact structure on the negative end, as desired.

\subsection{Almost symplectic structures on codimension 2 submanifolds}

Lemma 2.13. Let $\Sigma$ be a codimension 2 connected oriented submanifold of a $2 n$ dimensional almost symplectic manifold $(W, \eta)$. Then $\eta$ is homotopic to $\widetilde{\eta}$ for which $\Sigma$ is $\tilde{\eta}$-symplectic in the complement of a $(2 n-2)$-dimensional ball $D \subset \Sigma$. If $W$ is a manifold with boundary and $(\Sigma, \partial \Sigma) \subset(W, \partial W)$ a submanifold with non-empty boundary $\partial \Sigma$, then $\eta$ is homotopic to $\widetilde{\eta}$ for which $\Sigma$ is $\widetilde{\eta}$-symplectic everywhere.

Proof. Choose an almost complex structure $J$ compatible with $\eta$. It is an equivalent problem to deform $J$ into an almost complex structure $\widetilde{J}$ for which $T \Sigma$ is $\widetilde{J}$-invariant, because then for any almost symplectic structure $\widetilde{\eta}$ compatible with $\widetilde{J}$ the submanifold $\Sigma$ will be almost symplectic. We construct $\widetilde{J}$ inductively over cells of increasing dimension in some cell-decomposition of $\Sigma$. If $\partial \Sigma=\varnothing$ we assume that there is a unique $(2 n-2)$-cell. If $\partial \Sigma \neq \varnothing$ then $\Sigma$ can be isotoped into an arbitrarily small neighborhood of a $(2 n-3)$-dimensional complex $C \subset \Sigma$, and hence it suffices to perturb $J$ to $\widetilde{J}$ near the cells of $C$. Suppose that $\sigma \subset \Sigma$ is an $l$-dimensional cell, $l<2 n-2$, and that we already have deformed $J$ to make $\Sigma \widetilde{J}$-holomorphic near $\partial \sigma$. Let us choose two vector fields $e_{1}, e_{2}$ which trivialize the co-oriented normal bundle to $T \Sigma \subset T W$ on $\mathcal{O} p \sigma$. Furthermore, we can assume that $\widetilde{J} e_{1}=e_{2}$ on $\mathcal{O} p \partial \sigma$. We will arrange that $\widetilde{J} e_{1}=e_{2}$ on $\mathcal{O} p \sigma$. There is a homotopy $e_{2}^{t}, t \in[0,1]$, over $\mathcal{O} p \sigma$ and fixed on $\mathcal{O} p \partial \sigma$, such that $e_{2}^{0}=e_{2}, e_{2}^{1}=J e_{1}$, and so that $\left(e_{1}, e_{2}^{t}\right)$ remain linearly independent for all $t \in[0,1]$. Indeed, the obstruction to this lies in $\pi_{l}\left(S^{2 n-2}\right)=0$ for $l<2 n-2$. Let $R^{t}$ be a covering homotopy of orientation preserving automorphisms of $T W$ such that $R^{t}\left(e_{2}\right)=e_{2}^{t}$ and $R^{t}\left(e_{1}\right)=e_{1}$ for all $t \in[0,1]$. Let $J^{t}, t \in[0,1]$, be a 
homotopy of almost complex structures on $W$ such that

$-J^{0}=J$

- $J^{t}=J, t \in[0,1]$, in a neighborhood of the $(l-1)$-skeleton;

- $J^{t}=R_{*}^{t} J, t \in[0,1]$, on $T W_{\mathcal{O} p \sigma}$.

Then $\mathcal{O} p \sigma$ is $J^{1}$-holomorphic. Continuing this induction construction over all cells $\sigma$ of dimension $\leqslant 2 n-3$ we construct an almost complex structure $\widetilde{J}$ with the required properties.

\section{Proof of Theorem 1.1}

Corollary 2.12 implies that, if $n>2$, then it is sufficient to prove Theorem 1.1 in the case when the contact structure $\xi_{+}$is overtwisted on each of the components of $\partial_{+} W$, and for $n=2$ it is one of the assumptions that the contact structure $\xi_{+}$is overtwisted on at least one of the components of $\partial_{+} W$. Hence, Theorem 1.1 follows, even in a slightly stronger relative sutured cobordism version, from the following result.

Theorem 3.1. Let $\left(W, \partial_{-} W, \partial_{+} W\right)$ be a sutured cobordism of dimension $2 n \geqslant 4$ endowed with an almost symplectic structure $\eta$. Suppose that $\partial_{+} W, \partial_{-} W \neq \varnothing$. Let $\widetilde{\Lambda}$ be a Liouville form on $\mathcal{O} p \partial W$ such that the corresponding Liouville vector field $Z$ is inwardly transverse to $\partial_{-} W$ and outwardly transverse to $\partial_{+} W$. Denote $\xi_{ \pm}:=$ $\operatorname{Ker}\left(\left.\widetilde{\Lambda}\right|_{\partial_{ \pm} W}\right)$. Suppose that

$-\left.\eta\right|_{\mathcal{O} p W}=d \widetilde{\Lambda}$

- $\xi_{-}$is overtwisted on at least one of the components of $\partial_{-} W$;

- if $n>2$ then $\xi_{+}$is overtwisted on all of the connected components of $\partial_{+} W$, and if $n=2$ then $\xi_{+}$is overtwisted on at least one of the connected components of $\partial_{+} W$.

Then there exists a Liouville cobordism structure $\Lambda$ on $W$ such that

- $\operatorname{Ker}\left(\left.\Lambda\right|_{\partial_{ \pm} W}\right)=\xi_{ \pm}$;

- $d \Lambda$ is homotopic to $\eta$ through a homotopy of almost symplectic forms and the homotopy can be taken to be fixed on $\partial W$. 


\subsection{Reduction to handles}

We begin the proof of Theorem 3.1 with the following observation.

Lemma 3.2. Consider a cobordism $W$ obtained by concatenating two sutured cobordisms $W_{0}$ and $W_{1}$. Suppose that Theorem 3.1 holds for the cobordisms $W_{0}$ and $W_{1}$. Then it also holds for $W$ provided that $\left.\widetilde{\Lambda}\right|_{\partial_{+} W}$ is overtwisted on at least one of the components of $\partial_{+} W$ which intersects $\partial_{+} W_{1}$.

Proof. Recall that $\partial_{+} W=\partial_{+} W_{1} \cup\left(\partial_{+} W_{0} \backslash \partial_{-} W_{1}\right)$. One can modify the forms $\widetilde{\Lambda}$ and $\eta$ by an isotopy supported in $\mathcal{O} p \partial_{+} W$ to make $\left.\widetilde{\Lambda}\right|_{\partial_{+} W_{1}}$ overtwisted on at least one of the components of $\partial_{+} W_{1}$. Furthermore, we can deform the Liouville form $\tilde{\Lambda}$ on $\mathcal{O} p\left(\partial_{-} W_{1} \cap \partial_{+} W_{0}\right)$, so that it restricts to $\partial_{-} W_{1} \cap \partial_{+} W_{0}$ as an overtwisted contact form in the formal homotopy class of $\eta$, relative to $\left.\partial_{(} \partial_{+} W_{0} \backslash \partial_{-} W_{1}\right)$. We can then successively apply Theorem 3.1 to $W_{0}$ and to $W_{1}$ to get the required form $\Lambda$ on $W$.

Any cobordism $W$ with $\partial_{+} W \neq \varnothing$ can be presented as a result of successive handle attachments with handles of index $k=1, \ldots, 2 n-1$, starting with $\partial_{-} W$. If Morse $\left(W, \partial_{-} W\right) \leqslant n$ then Theorem 3.1 for $W$ follows from the Weinstein handlebody theory, see Theorem 2.8.

In general, the handles can be concatenated in the increasing index order. In particular, $W$ can be obtained by concatenating handles $W_{1}, \ldots, W_{j}$ of index $2 n-1$ to a cobordism $W^{\prime}$ with connected boundary $\partial_{+} W^{\prime}$. By rearranging these handles via handle slides, if necessary, we can assume that the form $\widetilde{\Lambda}$ is overtwisted on at least one of the components of $\partial_{+} W$ which intersects $\partial_{+} W_{i}$ for each $i=1, \ldots, j$. Hence, Lemma 3.2 implies that it is sufficient to prove Theorem 3.1 for handles. Moreover, the Weinstein handlebody theory, see Theorem 2.8 and Corollary 2.10. further reduces our task to proving Theorem 3.1 for handles of index $>n$.

We note, however, that the construction below works for all handles of index $k>1$, and in a slightly modified way for $k=1$ as well.

Lemma 3.3. Let $(W, \Lambda)$ be a sutured Liouville cobordism structure on a handle $W$ of index $k$, and let $\eta$ be an almost symplectic form which coincides with $d \Lambda$ on $\mathcal{O} p \partial W$. Suppose that the contact form $\left.\Lambda\right|_{\partial W}$ is overtwisted on at least one of the connected components of either $\partial_{-} W$ or $\partial_{+} W$. Then $W$ admits a Liouville cobordism structure $\widetilde{\Lambda}$ such that $d \widetilde{\Lambda}$ and $\eta$ are homotopic relative to $\partial W$. 
Proof. The space of almost symplectic structures is homotopy equivalent to the space of almost complex structures, and hence, the difference of the relative homotopy classes of $d \Lambda$ and $\eta$ can be viewed as an element $a \in \pi_{2 n}(S O(2 n) / U(n))$ (recall that $(W, \partial W)$ is homeomorphic to $\left.\left(D^{2 n}, S^{2 n-1}\right)\right)$. Considering $D^{2 n}$ as $D^{2 n-1} \times[0,1]$, the class $a$ can be viewed as a loop of $S O(2 n) / U(n)$-valued functions on $D^{2 n-1}$ which are constant on $\mathcal{O} p \partial D^{2 n-1}$. In turn, this loop can be viewed as a loop of almost contact structures on $D^{2 n-1}$.

Using Theorem 1.4 we can realize this loop as a loop of overtwisted contact forms $\alpha_{t}$ on $D^{2 n-1}, t \in[0,1]$, fixed on $\mathcal{O} p \partial D^{2 n-1}$. For a sufficiently large $C$ the form $\lambda:=e^{C t} \alpha_{t}$ defines a Liouville cobordism structure on the trivial cobordism $D^{2 n-1} \times[0,1]$. Let $P$ be a sutured version of this cobordism. By our assumptions, the contact form $\Lambda$ is overtwisted on one of the boundary components $V \subset \partial_{+} W$ or $V \subset \partial_{-} W$. Let us assume for determinacy that $V \subset \partial_{+} W$. Using Lemma 2.7, we construct a contact embedding $h:\left(\partial_{-} P, \operatorname{Ker} \lambda_{\partial_{-} P}\right) \rightarrow\left(V, \operatorname{Ker}\left(\left.\Lambda\right|_{V}\right)\right)$. Furthermore, using Lemma 2.5 we can adjust the Liouville form $\Lambda$ on $W$ to have $h^{*} \Lambda=\alpha_{0}$. Hence, we can use $h$ to construct a cobordism $\left(W^{\prime}, \Lambda^{\prime}\right)=(W, \Lambda) \underset{h}{\cup}(P, \lambda)$ by concatenating $P$ and $W$. There is an isotopy of $g_{t}: W \rightarrow W^{\prime}$ fixed outside $\mathcal{O} p \partial_{+} W \subset W^{\prime}$, moving points along trajectories of the Liouville field $Z^{\prime}$ corresponding to the Liouville form $\Lambda^{\prime}$, such that $g_{0}: W \hookrightarrow W^{\prime}$ is the inclusion and $g_{1}(W)=W^{\prime}$. Then the Liouville form $\widetilde{\Lambda}:=g_{1}^{*} \Lambda^{\prime}$ on $W$ has the required properties (i.e. we have "killed" the previous obstruction $\left.a \in \pi_{2 n}(S O(2 n) / U(n))\right)$. The proof for the case when an overtwisted component is contained in $\partial_{-} W$ works in a similar way with an inverse concatenation of $P$ and $W$ via a contact embedding $\partial_{+} P \rightarrow \partial_{-} W$.

\subsection{Beginning of the construction}

In what follows, we will be proving Theorem 3.1 in the case when $W$ is a handle of index $k>1$, i.e. $W=D^{k} \times D^{2 n-k}, \partial_{-} W=\partial D^{k} \times D^{2 n-k}, \partial_{+} W=D^{k} \times \partial D^{2 n-k}$. Moreover, in view of Lemma 3.3 it will be sufficient to construct the form $\Lambda$ without caring about the relative homotopy class of $d \Lambda$. The proof will be done by induction over $n$. The case $n=1$ is trivial. Suppose $n \geqslant 2$ and that the theorem is already established for cobordisms of dimension $<2 n$.

Let $\xi_{+}$be the contact structure $\operatorname{Ker}\left(\widetilde{\Lambda} \partial_{\partial_{+} W}\right)$ on $\partial_{+} W=D^{2 n-k} \times \partial D^{k}$. Assuming that $D^{k}$ and $D^{2 n-k}$ are discs of radius 1 , consider the equatorial disk $\Delta:=D^{k-1}\left(\frac{1}{2}\right) \subset D^{k}$ of radius $\frac{1}{2}$ and the codimension 2 submanifold $\Sigma:=\partial \Delta \times D^{2 n-k} \subset W$ with boundary $\partial \Sigma=\partial \Delta \times \partial D^{2 n-k} \subset \partial_{+} W$. There exists a diffeotopy $h_{t}: W \rightarrow W, t \in[0,1]$, such that $h$ is fixed on $\mathcal{O}_{p} \partial_{-} W, h_{0}=\operatorname{Id}$ and $h_{1}(\partial \Sigma)$ is a contact submanifold of $\left(\partial_{+} W, \xi_{+}\right)$ 
(which in the case $n=2$ means that $h_{1}(\partial \Sigma)$ is transverse to contact structure $\xi_{+}$). If $n>2$ or if $n=2$ and the index $k$ of the handle is $<3$ then we can also arrange that $\xi_{+}$is overtwisted on (every component of) the complement of $h_{1}(\partial \Sigma)$. Indeed, this follows from Theorem 1.4 and Lemma 2.7 , because $\left.\widetilde{\Lambda}\right|_{\partial_{+} W}$ is overtwisted on each of the components of $\partial_{+} W$. If $n=2$ and $k=3$ then we can isotope each that component of $h_{1}(\partial \Sigma)$ to a curve transverse to the contact structure $\operatorname{Ker}\left(\left.\widetilde{\Lambda}\right|_{\partial_{+} W}\right)$. To simplify the notation we will now write $\Sigma$ for the deformed submanifold $h_{1}(\Sigma)$.

The normal bundle to $\Sigma$ in $W$ is trivial, and, according to the contact normal neighborhood theorem, there exists a splitting $\widetilde{N}=\Sigma \times D^{2}$ of a tubular neighborhood of $\Sigma$ such that, if we define $\widetilde{M}_{\text {ext }}:=\widetilde{N} \cap \partial_{+} W=\partial \Sigma \times D^{2}$, then the contact structure $\xi_{+}$admits a contact form $\alpha_{+}$satisfying $\left.\alpha_{+}\right|_{\widetilde{M}_{\text {ext }}}=\delta+u d t$, where $\delta$ is a contact form on $\partial \Sigma$ and $(\sqrt{u}, t) \in([0,1] \times \mathbb{R} /(2 \pi \mathbb{Z}))$ are coordinates on $\tilde{N}$ induced from polar coordinates on the factor $D^{2}$. If $n>2$, we can also arrange that $\xi_{+}$is overtwisted on (every component of) the complement of $\widetilde{M}_{\text {ext }}$.

Lemma 3.4. The form dt extends from $\tilde{N} \backslash \Sigma$ to $W \backslash \Sigma$ as a closed 1 -form $\tau$ supported away from $\mathcal{O}_{p} \partial_{-} W$.

Proof. Note that $(\Sigma, \partial \Sigma)$ represents a trivial homology class in $H_{2 n-2}\left(W, \partial_{+} W\right)$. This implies that a circle $S:=x \times \partial D^{2}, x \in \Sigma$, represents a non-zero homology class in $H_{1}\left(W \backslash \Sigma, \partial_{-} W ; \mathbb{R}\right)$. Indeed, if there existed a relative chain $C$ in $\left(W \backslash \Sigma, \partial_{-} W\right)$ bounding $S$, then the union of $C$ with the normal fiber $x \times D^{2}$ would form a relative 2-cycle $\Gamma$ transversely intersecting $\Sigma$ at 1-point, and hence the Poincaré-dual cohomology class $P \Gamma \in H^{2 n-2}\left(W, \partial_{+} W\right)$ would evaluate non-trivially on $\Sigma$, contrary to the triviality of the homology class $[\Sigma] \in H_{2 n-2}\left(W, \partial_{+} W\right)$. Hence, there exists a cohomology class $a \in H^{1}\left(W \backslash \Sigma, \partial_{-} W ; \mathbb{R}\right)$ such that $a([S])=1$ and $\left.a\right|_{\Sigma \times y}=0$. Therefore, $\left.a\right|_{\tilde{N}}=[d t]$ and hence $d t$ extends as a closed 1-form $\tau:=a+d f$ for some smooth function $f$ vanishing on $\mathcal{O}_{p} \partial_{-} W$.

Using Lemma 2.13 we deform the almost symplectic structure $\eta$ to make $\left.\eta\right|_{\Sigma}$ almost symplectic.

\subsection{The case $n=2$}

Consider first the case $n=2$. For a sufficiently large constant $C$ the form $C \alpha_{+}-c d t$ is contact for all $c \in[0,1]$. Let us consider a smaller tubular neighborhood $N=\{u \leqslant$ $\left.\frac{1}{2 C}\right\} \subset \tilde{N}$. Denote $v=C u$, so that we have $N=\left\{v \leqslant \frac{1}{2}\right\}$ and $\left.C \alpha_{+}\right|_{N}=C \delta+v d t$. To simplify the notation we rename $C \alpha_{+}$and $C \delta$ back to $\alpha_{+}$and $\delta$. 
If $n=2$ then $\Sigma$ is a 2-dimensional surface with boundary, and hence, the form $\delta$ can be extended as a Liouville form $\lambda$ to $\Sigma$ such that the corresponding Liouville vector field transverse to $\partial \Sigma$ in the outward sense. Denote $M_{\text {ext }}:=\partial N \cap \partial_{+} W, M_{\text {int }}=$ $\partial N \backslash M_{\text {ext }}$, see Fig. 3.2 ,

Consider the Liouville 1-form $\mu:=(v-1) d t+\lambda$ on $N$. We have $\left.\mu\right|_{M_{\mathrm{ext}}}=\left.\left(\alpha_{+}-\tau\right)\right|_{M_{\mathrm{ext}}}$ and the Liouville field corresponding to $\mu$ points into $N$ along $M_{\text {int }}$, and out of $N$ along $M_{\text {ext }}$. Note that while $\mu$ blows up along $\Sigma$, the symplectic form $d \mu$ extends to $N$ as a symplectic form, and the extended form coincides with $d \lambda$ over $\Sigma$.

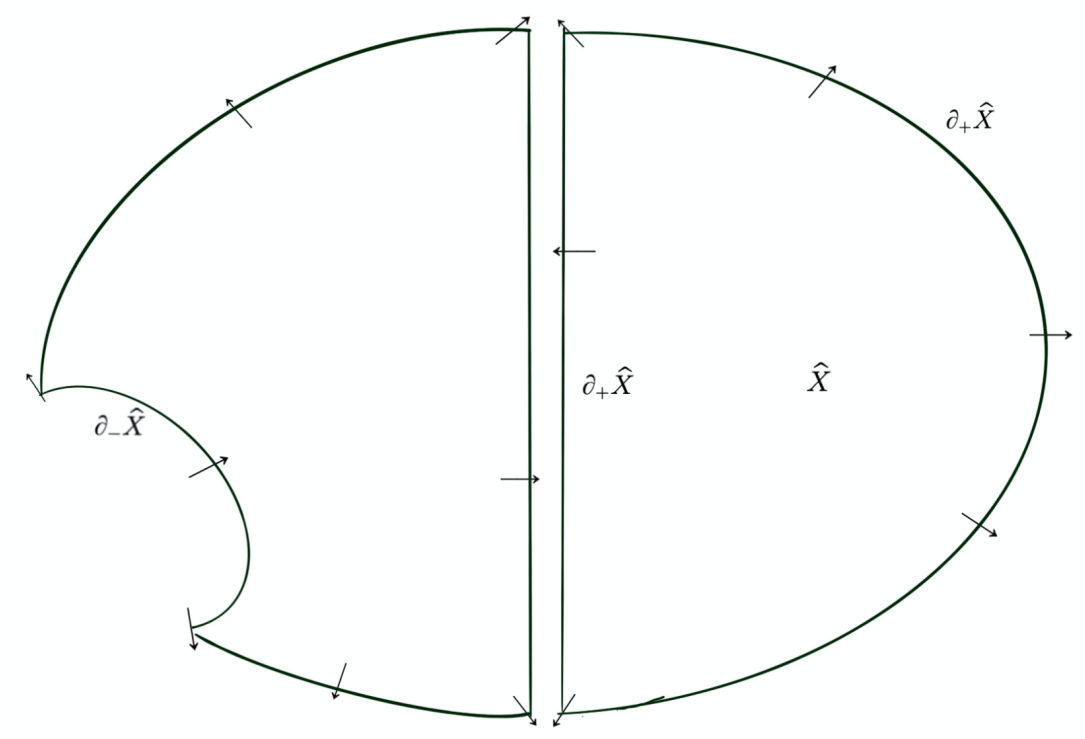

Fig. 3.1: Cobordism $\widehat{X}$

Consider the sutured cobordism

$$
\hat{X}=W \backslash N, \partial_{-} \hat{X}=\partial_{-} W, \partial_{+} \hat{X}=\left(\partial_{+} W \backslash M_{\text {ext }}\right) \cup M_{\text {int }},
$$

see Fig. 3.1.

Lemma 2.4, see Fig. 2.9, implies that $\operatorname{Morse}\left(\hat{X}, \partial_{-} \hat{X}\right)$ is equal to $14^{4}$ Consider a Liouville form $\widehat{\Lambda}$ on $\mathcal{O} p \partial_{+} \widehat{X}$ which restricts as $\alpha_{+}-\tau$ to $\partial_{+} W \backslash M_{\text {ext }}$ and which equals to $\mu$ on $M_{\text {int }}$.

\footnotetext{
${ }^{4}$ In the original version of the paper the Morse type of $\left(W \backslash \Sigma, \partial_{-} W\right)$ was stated incorrectly. We thank the anonymous referee for pointing out our mistake.
} 
By applying Theorem 2.8 we can extend $\widehat{\Lambda}$ to a Weinstein cobordism form $\widehat{\Lambda}$ on $\hat{X}$ which restricts as $f \alpha_{-}$to $\partial_{-} W$, and which is in the formal rel. $\partial_{-} W$ homotopy class of $\eta$. We can then extend $\widehat{\Lambda}$ to $W \backslash \Sigma$ as equal to $\mu$ on $N \backslash \Sigma$. Then the form $\Lambda=\widehat{\Lambda}+\tau$ is the required Liouville cobordism structure on $W$. Its relative to $\partial W$ homotopy class can be fixed using Lemma 3.3, and this concludes the proof of Theorem 3.1 in the case $n=2$.

\subsection{Case $n>2$}

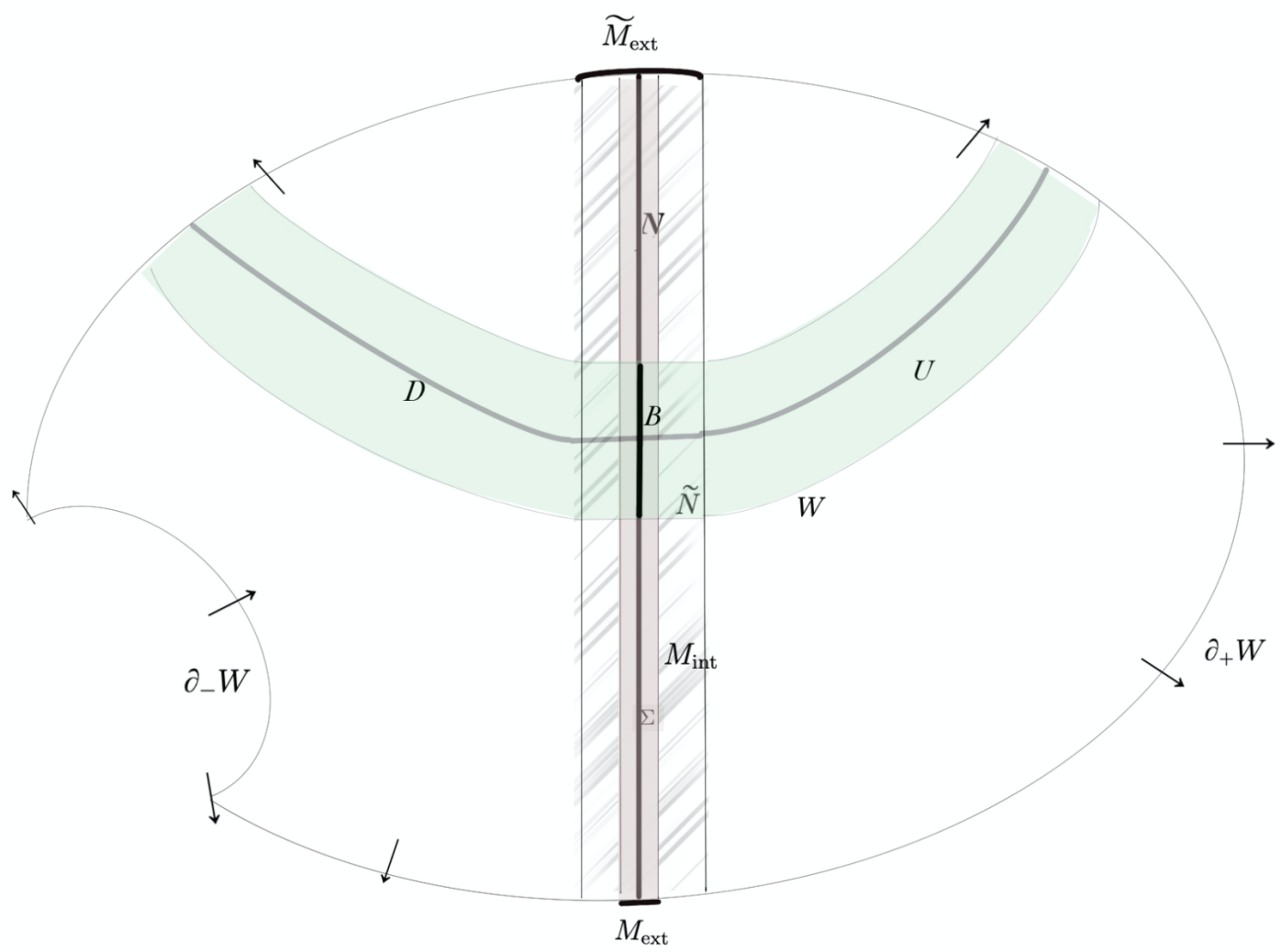

Fig. 3.2: Hypersurface $\Sigma$, disc $D$, and their neighborhoods

Suppose $n>2$. Choose an embedded ball $B \Subset \operatorname{Int} \Sigma$ centered at a point $p \in \Sigma$ and consider an overtwisted contact form $\beta$ on $\partial B$ in the formal homotopy class 
determined by $\eta$. Let $(D, \partial D) \subset(W, \partial W)$ be an embedded 2-disc such that $D \cap \tilde{N}=$ $p \times D^{2}$. We extend the polar coordinates $(\sqrt{u}, t)$ to the disc $D$ such that $\left.u\right|_{\partial D}=K$, where the constant $K>1$ will be chosen later.

Let $U=D \times B$ be a split tubular neighborhood of $D$ such that $U \cap \Sigma=B$ where $B \subset \Sigma$ is the ball chosen above, and the splittings of $U$ and $\tilde{N}$ agree on $\tilde{N} \cap U$, see Fig. 3.2. We also assume that $B \times \partial D \subset \partial_{+} W$. Choosing a radial coordinate $s$ on $B$, we extend the coordinates $s$ and $(u, t)$ to $U$ by pulling them back to $U=D \times B$ via projections to the second and first factors, respectively. For $\sigma \in(0,1]$ we denote

$$
U^{\sigma}:=\{s<\sigma\}, H_{\mathrm{ext}}^{\sigma}=\partial U_{\sigma} \cap \partial_{+} W, H_{\mathrm{int}}^{\sigma}:=\partial U \backslash H_{\mathrm{ext}}^{\sigma}
$$

and will write $H_{\text {ext }}$ and $H_{\text {int }}$ instead of $H_{\text {ext }}^{1}$ and $H_{\text {int }}^{1}$, see Fig. 3.3 .

The closed forms $\tau$ and $d t$ on $U$ are in the same cohomology class, and hence we can modify $\tau$ to make it equal to $d t$ on $(N \cup U) \backslash \Sigma$.

Lemma 3.5. There exists a contact form $\alpha$ on $\partial_{+} W \backslash \partial D$ with the following properties:

(i) $\alpha=C \alpha_{+}$on $\widetilde{M}_{\mathrm{ext}} \cup \mathcal{O} p \partial^{2} W$ for a constant $C \geqslant 1$; in particular, $\left.\alpha\right|_{\widetilde{M}_{+}}=$ $C u d t+C \delta$

(ii) the contact structures Ker $\alpha$ and $\left.\xi_{+}\right|_{\partial_{+} W \backslash \partial D}$ are homotopic relative $\widetilde{M}_{\text {ext }} \cup \mathcal{O} p \partial^{2} W$;

(iii) $\left.\alpha\right|_{H_{\text {ext }}^{\frac{1}{2}} \backslash \partial D}=2 d t+s \beta$

(iv) $\alpha-c \tau$ is contact for any $c \in[0,1]$.

Proof. Thanks to our choice of $\beta$ on $\partial B$ in the formal homotopy class determined by $\eta$, we can use Theorem 1.4 to construct a contact form $\alpha^{\prime}$ on $\partial_{+} W \backslash \partial D$ which satisfies conditions (i) and (ii) and which is equal to $2 d t+s \beta$ on $H_{\text {ext }} \backslash \partial D$. By multiplying $\alpha^{\prime}$ by a sufficiently large constant $C$ we can ensure that $C \alpha^{\prime}-c \tau$ is contact for all $c \in[0,1]$. We claim that there exists a $C^{\infty}$-function $h:[0,1] \rightarrow[1, C]$ such that

$\dagger h(s)=1$ for $s \in\left[0, \frac{1}{2}\right]$

$\dagger h(s)=C$ near $s=1$ and

$\dagger$ the form $h(s)(2 d t+s \beta)-c d t$ is contact on $H_{\text {ext }} \backslash \partial D$ for all $c \in[0,1]$. 
Indeed, let us first observe that it is sufficient to verify the latter condition for $c=1$. Indeed, let $R$ be the Reeb vector field of the form $h(s)(2 d t+s \beta)$. The form $h(s)(2 d t+s \beta)-c d t$ is contact if $c d t(R) \neq 1$ everywhere in $H_{\text {ext }} \backslash \partial D$. But on $H_{\text {ext }}^{\frac{1}{2}} \backslash \partial D$ we have $R=\frac{1}{2} \frac{\partial}{\partial t}$ and $d t(R)=\frac{1}{2}$. Hence, if the form $h(s)(2 d t+s \beta)-d t$ is contact everywhere then $d t(R)<1$, and thus, $c d t(R)<1$ as well. The contact condition for the form $h(s)(2 d t+s \beta)-d t=(2 h(s)-1) d t+s h(s) \beta$ is equivalent to the inequality $\frac{d}{d s}\left(\frac{s h(s)}{2 h(s)-1}\right)>0$. Take a $C^{\infty}$-function $\psi:[0,1] \rightarrow \mathbb{R}$ such that $\psi(s)=s$ for $s \in\left[0, \frac{1}{2}\right]$, $\psi(s)=\frac{C s}{2 C-1}$ near $s=1$, and which satisfies inequalities $2 \psi(s)>s$ and $\psi^{\prime}(s)>0$ for all $s \in[0,1]$. Then the function $h(s)=\frac{\psi(s)}{2 \psi(s)-s}$ has the required properties. Indeed, we have $h(s)=1$ for $s \in\left[0, \frac{1}{2}\right]$ and $h(s)=C$ near $s=1$. We also have $\frac{s h(s)}{2 h-1}=\psi(s)$, and hence the form

$$
h(s)(2 d t+s \beta)-d t=(2 h(s)-1) d t+\frac{s h(s)}{2 h(s)-1} \beta=(2 h(s)-1)(d t+\psi \beta)
$$

is contact because $\psi^{\prime}(s)>0$. Hence, the form

$$
\alpha:= \begin{cases}h(s)(2 d t+s \beta), & \text { on } H_{\text {ext }} \backslash \partial D ; \\ C \alpha^{\prime}, & \text { on } \partial_{+} W \backslash H_{\text {ext }}\end{cases}
$$

has the required properties.

If the constant $C$ in Lemma 3.5 is chosen large enough then the induction hypothesis allows us to find a Liouville form $\lambda$ on $\Sigma \backslash p$ with a conical singularity at $p$, and such that

- $\left.\lambda\right|_{\partial \Sigma}=C \delta$

- the Liouville field of $\lambda$ is outwardly transverse to $\partial \Sigma$;

- $\left.\lambda\right|_{B}=s \beta$ and

- $\left.d \lambda\right|_{\Sigma \backslash B}$ is in the almost symplectic homotopy class of $\eta$.

We now fix the constant $K=\left.u\right|_{\partial D}$ to be equal to $2 C$. Denote

$$
\begin{aligned}
& v:=C u, N:=\left\{v<\frac{1}{2}\right\} \subset \tilde{N}, M_{\text {int }}:=\left\{v=\frac{1}{2}\right\}, M_{\text {ext }}=N \cap \partial_{+} W, \\
& P:=\left\{\max \left(1-v, \frac{v-1}{2}\right) \leqslant s \leqslant \frac{1}{2}\right\} .
\end{aligned}
$$

We have $\partial N=M_{\text {int }} \cup M_{\text {ext }}$ and $H_{\text {ext }}=\{v=2, s<1\}$. 


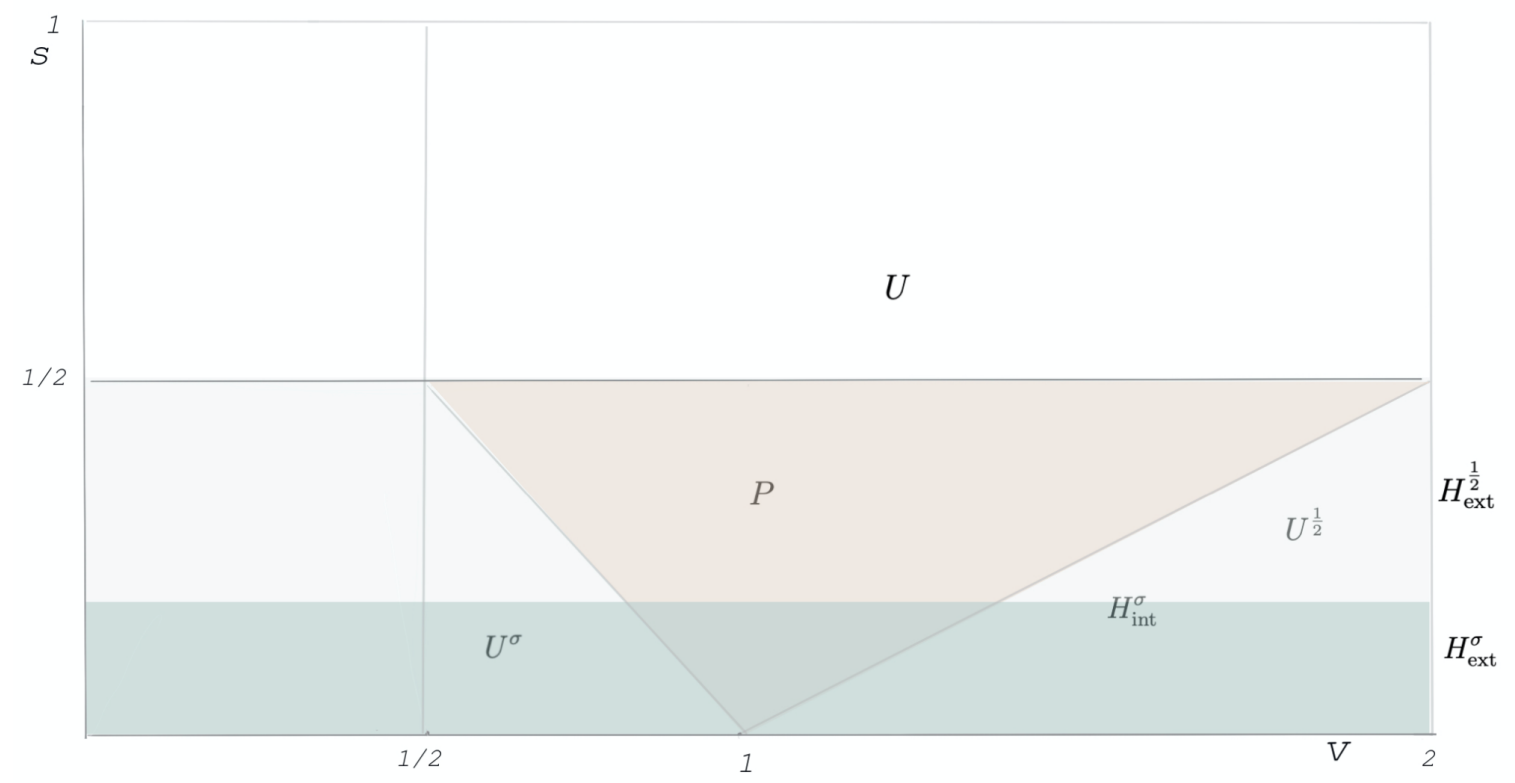

Fig. 3.3: The inside of the neighborhood $U$

Proposition 3.6. For a sufficiently small $\sigma \in\left(0, \frac{1}{2}\right)$ the sutured cobordism

$$
\left(X^{\sigma}:=W \backslash U^{\sigma}, \partial_{-} X^{\sigma}:=\partial_{-} W \cup H_{\text {int }}^{\sigma}, \partial_{+} X^{\sigma}:=\partial_{+} W \backslash H_{\mathrm{ext}}^{\sigma}\right)
$$

admits a Liouville cobordism structure $\Lambda^{\sigma}$ such that

- $\left.\Lambda^{\sigma}\right|_{\mathcal{O} p H_{\text {int }}^{\sigma}}=s \beta+v d t$

- $\left.\Lambda^{\sigma}\right|_{\partial_{+} X^{\sigma}}=\alpha$;

- $\operatorname{Ker}\left(\left.\Lambda^{\sigma}\right|_{\partial_{-} W}\right)=\xi_{-}$.

Before proving this proposition we deduce from it Theorem 3.1 .

Deducing Theorem 3.1 from Proposition 3.6. Using Theorem 1.4 we can extend $\Lambda^{\sigma}$ to a neighborhood $\Omega \supset H_{\text {ext }}^{\sigma}$ so that $\left.\Lambda^{\sigma}\right|_{\partial_{+} W}$ is a contact form and the contact structure $\operatorname{Ker}\left(\left.\Lambda^{\sigma}\right|_{\partial_{+} W}\right)$ on $\partial_{+} W$ is homotopic to the contact structure $\xi_{+}$. Take an embedded $2 n$-ball $Q \subset \operatorname{Int} U^{\sigma}$ with boundary $\partial Q \subset \partial U^{\sigma} \cup \Omega$ transverse to the 


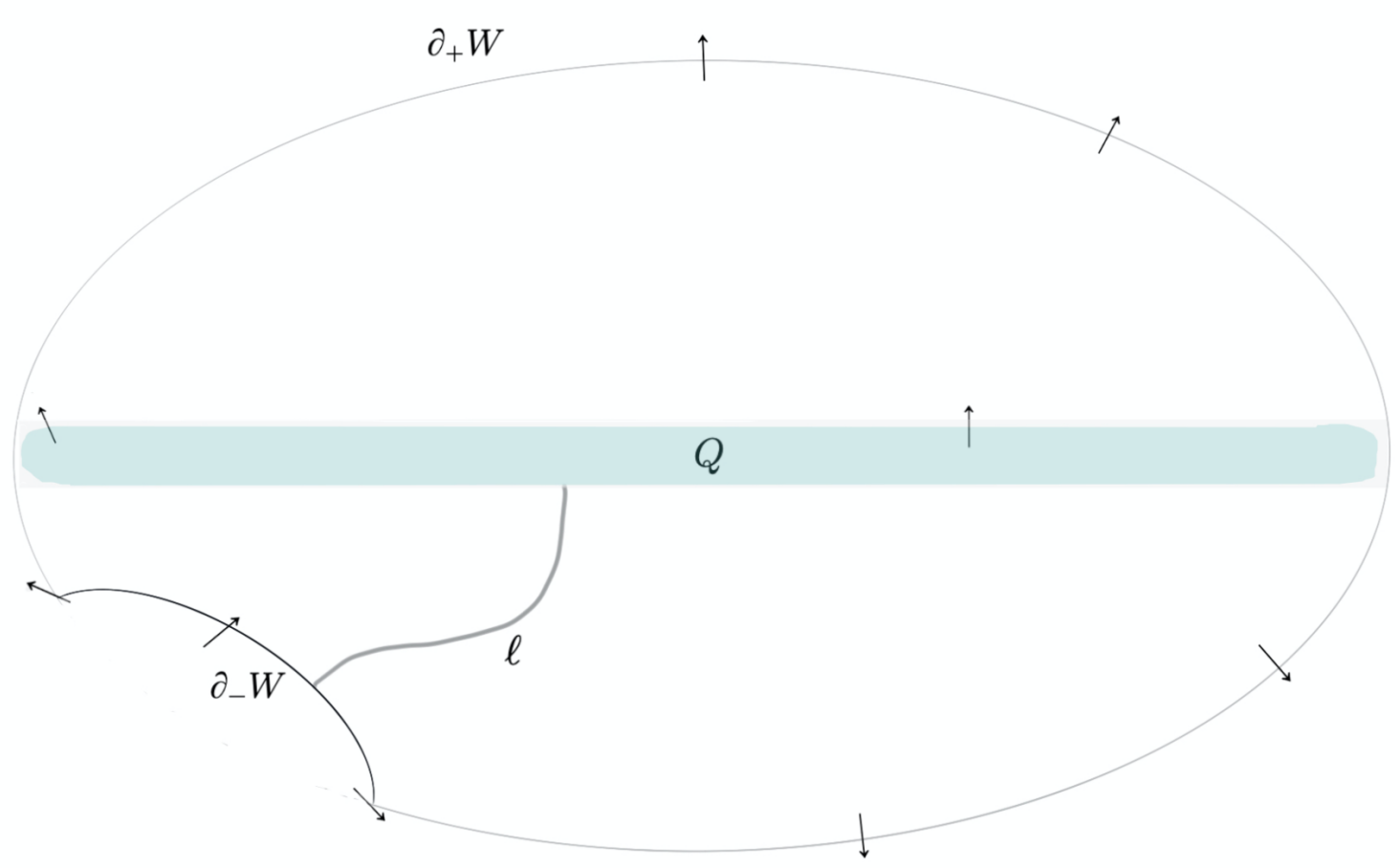

Fig. 3.4: Connect summing of $\partial_{-} W$ and $Q$

Liouville field of the form $\Lambda^{\sigma}$. The extended this way form $\Lambda^{\sigma}$ defines a Liouville cobordism structure on $W^{\prime}:=W \backslash \operatorname{Int} Q$ with $\partial_{-} W^{\prime}=\partial_{-} W \cup \partial Q, \partial_{+} W^{\prime}=\partial_{+} W$.

Our next goal is to modify the cobordism $W^{\prime}$ by connect summing $\partial_{-} W$ and $\partial Q$, see Fig. 3.4. To do that, choose an embedded arc $\ell$ connecting $\partial_{-} W$ with $\partial Q$, which coincides near $\partial \ell$ with a flow line of the Liouville field of $\Lambda^{\sigma}$ and such that $\int_{\ell} \Lambda^{\sigma}=0$. We can modify the form $\Lambda^{\sigma}$ by adding an exact form $d H$ supported in Int $W$ to make it vanishing on $\ell$. By subtracting from $W^{\prime}$ the Weinstein handle $H_{1}$ with the core disc $\ell$, see Section 2.3, we get a Liouville cobordism $W^{\prime \prime}$ whose new negative boundary is the connected sum $\partial_{-} W \# \partial Q$ along $\ell$. Finally we note that there exists a fixed near $\partial_{+} W$ isotopy $\rho_{\tau}: W^{\prime} \rightarrow W, \tau \in[0,1]$, such that $\rho_{0}=\operatorname{Id}$ and $\rho_{1}(W)=W^{\prime \prime}$. The pull-back Liouville form $\Lambda:=\rho_{1}^{*}\left(\Lambda^{\sigma}\right)$ is a Liouville cobordism structure on $W$ which restricts to $\partial_{ \pm} W$ as an overtwisted contact form in the formal homotopy class of $\xi_{ \pm}$, and hence, in view of Theorem 1.4 , the contact structure $\operatorname{Ker}\left(\left.\Lambda\right|_{\partial_{ \pm}}\right) W$ is isotopic to $\xi_{ \pm} \cdot$ 


\subsection{Proof of Proposition 3.6}

Lemma 3.7. Let $\left(X, \partial_{-} X, \partial_{+} X\right)$ be the sutured cobordism

$$
\begin{aligned}
& X:=W \backslash\left(U^{\sigma} \cup N\right), \\
& \partial_{-} X:=\left(H_{\mathrm{int}}^{\frac{1}{2}} \backslash N\right) \cup \partial_{-} W, \quad \partial_{+} X:=\left(\partial_{+} W \backslash M_{\mathrm{ext}}\right) \cup\left(M_{\mathrm{int}} \backslash U^{f r a c 12}\right) .
\end{aligned}
$$

Then $\operatorname{Morse}\left(X, \partial_{-} X\right) \leqslant 3$.

Proof. As in the case $n=2$, Lemma 2.4 implies that $\operatorname{Morse}\left(\hat{X}, \partial_{-} \hat{X}\right)=1$ for the sutured cobordism $\widehat{X}=W \backslash N, \partial_{-} \hat{X}=\partial_{-} W, \partial_{+} \widehat{X}=\left(\partial_{+} W \backslash M_{\text {ext }}\right) \cup M_{\text {int }}$, see Fig. 2.9 and Fig. 3.5.

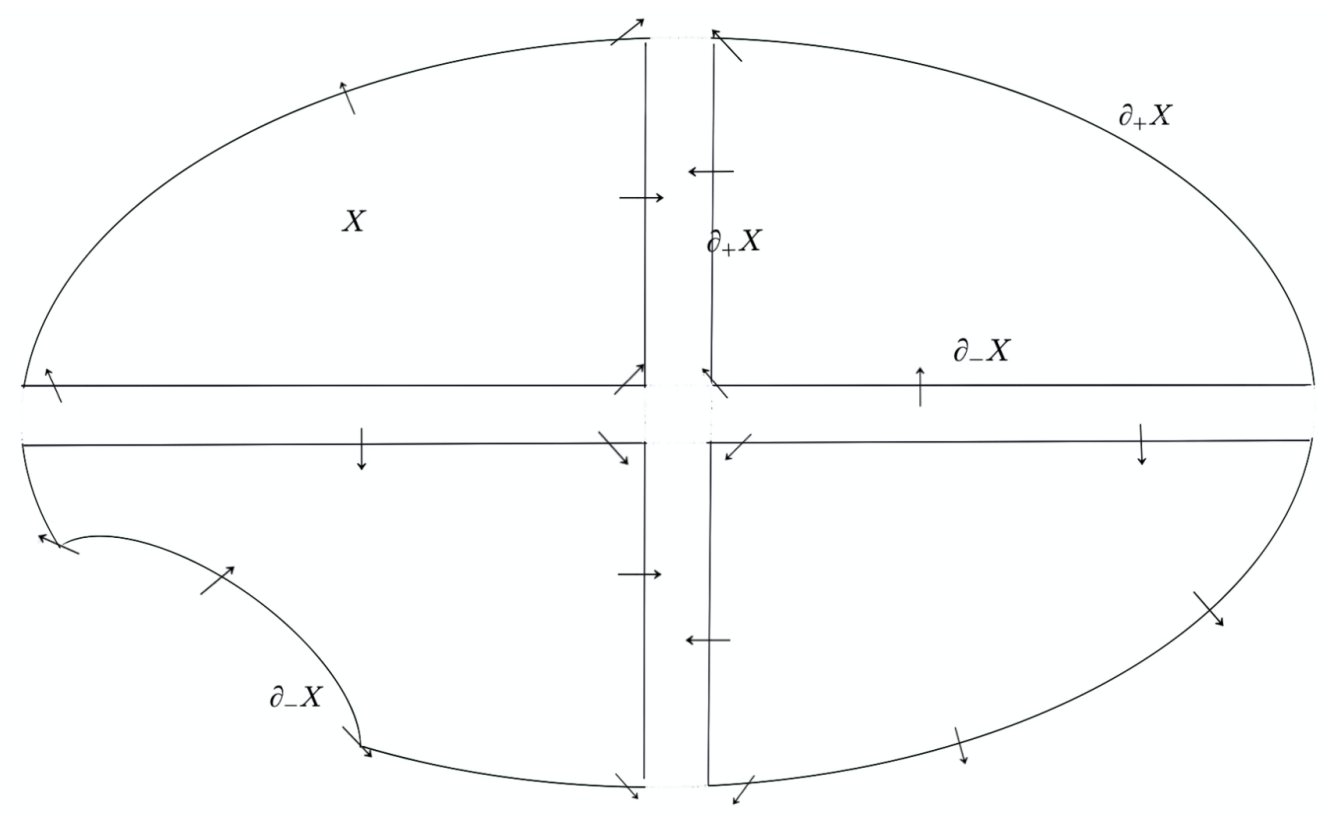

Fig. 3.5: Cobordism X

Hence the pair $\left(\hat{X}, \partial_{+} \hat{X}\right)$ is $(2 n-2)$-connected. Taking into account that $n \geqslant 3$ we conclude that the annulus $A=\left\{\frac{1}{2} \leqslant v \leqslant 2, s=0\right\} \subset D$ is isotopic in $\hat{X}$ relative $\partial A$ to an annulus contained in $\partial_{+} \hat{X}$. Moreover, there exists an embedding of the sutured version $\widehat{A}$ of the trivial cobordism $A \times I$ into $\widehat{X}$ such that $\partial_{-} \widehat{A}=A$ and 
$\partial_{+} \widehat{A} \subset \partial_{+} \widehat{X}$. Indeed, the general position argument provides us with an embedding if $n>3$ and an immersion if $n=3$. But any self-intersection point can be pushed to the boundary, i.e. one can get rid of the self-intersection points by changing the embedding class of the boundary $\partial_{+} \widehat{A} \subset \partial_{+} W$.

The cobordism $\left(X, \partial_{-} X, \partial_{+} X\right)$ can be obtained from the cobordism $\left(\hat{X}, \partial_{+} \hat{X}, \partial_{-} \hat{X}\right)$ by removing a tubular neighborhood of $A$ and adjoining the boundary of this neighborhood to $\partial_{+} X$, as it described in Lemma 2.3. Hence, we can apply that lemma to conclude that

$$
\operatorname{Morse}\left(X, \partial_{-} X\right) \leqslant \max \left(\operatorname{Morse}\left(\widehat{X}, \partial_{-} \hat{X}\right), \operatorname{Morse}(A)+1\right)=3
$$

Proof of Proposition 3.6. Consider the form $\mu$ on $(U \backslash D) \cup(N \backslash \Sigma)$ which is equal to $\lambda+(v-1) d t$ on $N \backslash \Sigma$ and equal to $s \beta+(v-1) d t$ on $U \backslash D$. Lemma 3.7 allows us to apply Corollary 2.10 to construct a sutured Liouville cobordism structure $\widehat{\Lambda}$ on $\left(X, \partial_{-} X, \partial_{+} X\right)$ such that

- $\widehat{\Lambda}=\mu$ on $\mathcal{O} p(\partial N \cap X)$

- $\operatorname{Ker}\left(\left.\widehat{\Lambda}\right|_{\partial_{-} W}\right)=\xi_{-}$

$-\left.\widehat{\Lambda}\right|_{\partial_{+} W \backslash\left(M_{\text {ext }} \cup H_{\text {ext }}^{\frac{1}{2}}\right)}=\alpha-\tau$

$-\left.\widehat{\Lambda}\right|_{H_{\mathrm{int}}^{\frac{1}{2}}}=\phi \mu$ for a function $\phi: H_{\mathrm{int}}^{\frac{1}{2}} \rightarrow(0,1]$ such that $\left.\phi\right|_{\mathcal{O}_{p} \partial H_{\mathrm{int}}^{\frac{1}{2}}}=1$.

Note that the Liouville vector field $Z$ corresponding to the form $\mu=(v-1) d t+s \beta$ on $U \backslash D$ is equal to $s \frac{\partial}{\partial s}+(v-1) \frac{\partial}{\partial v}$, and therefore, its negative flow flow $Z^{\nu}, \nu \leqslant 0$ is given by the formula

$$
Z^{\nu}(v, s)=\left(1+e^{\nu}(v-1), e^{\nu} s\right), \quad \nu \leqslant 0,
$$

and hence, it leaves invariant the cone $P:=\left\{\max \left(1-u, \frac{u-1}{2}\right) \leqslant s \leqslant \frac{1}{2}\right\}$, see Fig. 3.3 . Define an isotopy

$$
\alpha_{\nu}: H_{\mathrm{int}}^{\frac{1}{2}} \rightarrow P, \nu \in[0,1],
$$

by the formula

$$
\alpha_{\nu}(x)=Z^{\nu \ln \phi(x)}, x \in H_{\mathrm{int}}, \nu \in[0,1] .
$$


We have $\alpha_{0}=\mathrm{Id}, \alpha_{1}^{*} \mu=\phi \mu$ and $\alpha_{\nu}\left(H_{\text {int }}\right) \cap \overline{U^{\sigma}}=\varnothing$ for a sufficiently small $\sigma>0$ and all $\nu \in[0,1]$.

Let us extend the isotopy $\alpha_{\nu}$ to an isotopy $\bar{\alpha}_{\nu}: X \rightarrow X^{\sigma}=W \backslash U^{\sigma}$ which is fixed on $(\mathcal{O} p \partial W) \cap X$. Note that the push-forward form $\left(\bar{\alpha}_{1}\right)_{*} \widehat{\Lambda}$ coincides with $\mu$ on $\mathcal{O} p \alpha_{1}\left(H_{\text {int }}\right)$. Hence, we can define a Liouville form $\bar{\Lambda}$ on $X^{\sigma}=W \backslash\left(U^{\sigma} \cup \Sigma\right)$ by setting it equal to $\left(\bar{\alpha}_{1}\right)_{*} \widehat{\Lambda}$ on $\bar{\alpha}_{1}(X)$, and equal to $\mu$ elsewhere on $X^{\sigma} \backslash \Sigma$. It remains to observe that the form $\Lambda^{\sigma}:=\bar{\Lambda}+\tau$ extends smoothly to $X^{\sigma}$ and has the required properties. This concludes the proof of Proposition 3.6.

\section{References}

[1] M. S. Borman, Y. Eliashberg and E. Murphy, Existence and classification of overtwisted contact structures in all dimensions, Acta Math. 215(2015), 281361.

[2] R. Casals, E. Murphy, and F. Presas, Geometric criteria for overtwistedness, J. Amer. Math. Soc., 32(2019), 563-604.

[3] K. Cieliebak and Y. Eliashberg, From Stein to Weinstein and Back - Symplectic Geometry of Affine Complex Manifolds, Colloquium Publications Vol. 59, Amer. Math. Soc. (2012).

[4] Y. Eliashberg, Classification of overtwisted contact structures on 3-manifolds, Invent. Math. 98 (1989), no. 3, 623-637.

[5] Y. Eliashberg, Topological characterization of Stein manifolds of dimension $>2$, Internat. J. Math. 1, no. 1, 29-46 (1990).

[6] Y. Eliashberg, Filling by holomorphic discs and its applications, London Math. Soc. Lect. Notes, 151(1991), 45-68.

[7] Y. Eliashberg, Weinstein manifolds revisited, Proc. Sympos. Pure Math., 99(2019), Amer. Math. Soc., Providence, RI, 2018, pp. 59-82.

[8] Y. Eliashberg, A few remarks about symplectic filling, Geom. Topol., 8(2004), 277-293.

[9] Y. Eliashberg, M Gromov, Convex symplectic manifolds, Proc. Symp. Pure Math., 52(1991), Amer. Math. Soc., Providence, RI, 135-162, 
[10] Y. Eliashberg, E. Murphy, Lagrangian caps, Geom. Funct. Anal, 23(2013), 14831514.

[11] J. Etnyre, On Symplectic Fillings, Algebr. Geom. Topol., 4(2004), 73-80.

[12] J. Etnyre, K. Honda, On symplectic cobordisms, Math. Annal., 323(2002), 3139.

[13] D. Gay, Four-dimensional symplectic cobordisms containing three-handles, Geom. Topol., 10(2006), 1749-1759.

[14] H. Geiges, Symplectic manifolds with disconnected boundary of contact type, Int. Math. Res. Notices 1(1994), 23-30.

[15] J. Gray, Some global properties of contact structures, Ann. of Math. (2) 69(1959), 421-450.

[16] M. Gromov, Pseudo holomorphic curves in symplectic manifolds, Invent. Math., 82(1985), 307-347.

[17] P. Kronheimer, T. Mrowka, Monopoles and three-manifolds, New Mathematical Monographs, 10, Cambridge University Press, Cambridge, 2007.

[18] D. McDuff, Symplectic manifolds with contact type boundary, Invent. Math. 103(1991), 651-671.

[19] P. Massot, K. Niederkrüger, C. Wendl, Weak and strong fillability of higher dimensional contact manifolds, Invent. Math., 192(2013), 287-373.

[20] D. McDuff, Symplectic manifolds with contact type boundaries, Invent. Math. 103(1991), 651-671.

[21] J. Milnor, On a cobordism ring $\Omega^{*}$ and a complex analogue, Part I, Amer. J. Math., 82(1960), 505-521.

[22] Y. Mitsumatsu, Anosov flows and non-stein symplectic manifolds, Ann. Inst. Fourier Grenoble, 45(1995), 1407-1421.

[23] T. Mrowka, Y. Rollin, Legendrian knots and monopoles. Algebr. Geom. Topol. 6 (2006), 1-69.

[24] E. Murphy, Loose Legendrian embeddings in high dimensional contact manifolds, arXiv:1201.2245. 
[25] S.P. Novikov, Some problems in the topology of manifolds connected with the theory of Thom spaces, Soviet Math. Dokl., 1(1960), 717-720.

[26] K. Niederkrüger, The plastikstuffe a generalization of overtwisted disc to higher dimensions, Algebr. Geom. Topol. 6(2006), 2473- 2508.

[27] A. Weinstein, Contact surgery and symplectic handlebodies, Hokkaido Math. J. 20(1991), 241-251.

[28] C. Wendl, Non-exact symplectic cobordisms between contact 3-manifolds, J. Diff. Geom. 95(2013), 121-182.

[29] C. Wendl, https://symplecticfieldtheorist.wordpress.com/author/lmpshd/. 